\title{
ANALYSIS OF FINITE ELEMENT APPROXIMATIONS OF STOKES EQUATIONS WITH NON-SMOOTH DATA
}

\author{
RICARDO G. DURÁN, LUCIA GASTALDI, AND ARIEL L. LOMBARDI
}

\begin{abstract}
In this paper we analyze the finite element approximation of the Stokes equations with non-smooth Dirichlet boundary data. To define the discrete solution, we first approximate the boundary datum by a smooth one and then apply a standard finite element method to the regularized problem.

We prove almost optimal order error estimates for two regularization procedures in the case of general data in fractional order Sobolev spaces, and for the Lagrange interpolation (with appropriate modifications at the discontinuities) for piecewise smooth data. Our results apply in particular to the classic lid-driven cavity problem improving the error estimates obtained in 9].

Finally, we introduce and analyze an a posteriori error estimator. We prove its reliability and efficiency, and show some numerical examples which suggest that optimal order of convergence is obtained by an adaptive procedure based on our estimator.
\end{abstract}

\section{INTRODUCTION}

The goal of this paper is to analyze finite element approximations of the Stokes equations with non smooth Dirichlet boundary data. For the Laplace equation the analogous problem has been analyzed in recent years in [4, 5.

Before explaining the problem and goals let us introduce some notation. For $s$ a real number, $1 \leq p \leq \infty$, and $D$ a domain in $\mathbb{R}^{d}$ or its boundary or some part of it, we denote by $W^{s, p}(D)$ the Sobolev space on $D$, and by $\|\cdot\|_{s, p, D}$ and $|\cdot|_{s, p, D}$ its norm and seminorm respectively (see, for example, 1, 2]). As it is usual, we write $H^{s}(D)=W^{s, 2}(D)$ and omit the $p$ in the norm and seminorm when it is 2 . Moreover, bold characters denote vector valued functions and the corresponding functional spaces. The notation $(\cdot, \cdot)_{D}$ stands for the scalar product in $L^{2}(D)$ as well as for the duality pairing between a Sobolev space and its dual; when no confusion may arise the subscript indicating the domain is dropped.

The subspace of $H^{1}(D)$ with zero trace on the boundary is denoted as usual by $H_{0}^{1}(D)$, while $L_{0}^{2}(D)$ is the subspace of $L^{2}(D)$ of functions with zero mean value.

1991 Mathematics Subject Classification. 65N30, 65N15.

Key words and phrases. Stokes equations, finite elements, non smooth data, a posteriori error analysis.

The first and third authors are supported by ANPCyT under grant PICT2014-1771, by CONICET under grant PIP $11220130100184 \mathrm{CO}$ and by Universidad de Buenos Aires under grant 20020160100144BA. Second author gratefully acknowledges the hospitality of University of Buenos Aires (Department of Mathematics) during her visit on March 2017 under the project SAC.AD002.001.003/ ARGENTINA - CONICET - 050.000. She is partially funded by IMATICNR and GNCS-INDAM. Third author acknowledges IMATI (Pavia) and DICATAM, University of Brescia, for the hospitality during his visit in November 2018 supported by the bilateral project CONICET (Argentina) - CNR (Italy) and FLR 2015/2016 (University of Brescia). He is also supported by Universidad Nacional de Rosario under grant ING568. 
Let $\Omega \subset \mathbf{R}^{d}, d=2,3$, be a Lipschitz domain with boundary $\Gamma=\partial \Omega$ and denote by $\mathbf{n}$ the outward unit vector normal to the boundary.

We consider the Stokes problem

$$
\begin{array}{rlrl}
-\Delta \mathbf{u}+\nabla p & =\mathbf{f} & & \text { in } \Omega \\
\operatorname{div} \mathbf{u}=\eta & & \text { in } \Omega \\
\mathbf{u}=\mathbf{g} & & \text { on } \Gamma .
\end{array}
$$

where $\mathbf{f}, \eta$ and $\mathbf{g}$ are given data. If $\mathbf{f} \in \mathbf{H}^{-1}(\Omega), \eta \in L^{2}(\Omega), \mathbf{g} \in \mathbf{H}^{1 / 2}(\Gamma)$, and the compatibility condition

$$
\int_{\Gamma} \mathbf{g} \cdot \mathbf{n}=\int_{\Omega} \eta
$$

is satisfied, existence and uniqueness of solution $\mathbf{u} \in \mathbf{H}^{1}(\Omega)$ and $p \in L^{2}(\Omega) / \mathbb{R}$ is a well known result (see for example [21, Page 31]). Moreover, the following a priori estimate holds true,

$$
\|\mathbf{u}\|_{1, \Omega}+\|p\|_{L^{2}(\Omega) / \mathbb{R}} \leq C\left(\|\mathbf{f}\|_{-1, \Omega}+\|\eta\|_{0, \Omega}+\|\mathbf{g}\|_{1 / 2, \Gamma}\right) .
$$

The classic analysis of finite element methods for this problem is based on the variational formulation working with the spaces $\mathbf{H}^{1}(\Omega)$ for the velocity $\mathbf{u}$ and $L^{2}(\Omega)$ for the pressure $p$. If $\mathbf{g} \notin \mathbf{H}^{1 / 2}(\Gamma)$ then the solution $\mathbf{u} \notin \mathbf{H}^{1}(\Omega)$, and therefore, that theory cannot be applied. This situation arises in many practical situations. A typical example is the so called lid-driven cavity problem where $\Omega$ is a square and the boundary velocity $\mathbf{g}$ is a piecewise constant vector field which has jumps at two of the vertices, and therefore, does not belong to $\mathbf{H}^{1 / 2}(\Gamma)$. However, this example is used in many papers as a model problem to test finite element methods using some regularization of $\mathbf{g}$ (although many times how the boundary condition is treated is not clearly explained). Error estimates for this particular case were obtained in 9, 16. In [9, the authors work with $L^{p}$ based norms and use that $\mathbf{u} \in \mathbf{W}^{1, p}(\Omega)$ for $1<p<2$. In [16] a particular regularization of the boundary datum is considered.

More generally, we will consider boundary data $\mathbf{g} \in \mathbf{L}^{2}(\Gamma)$ using some regularization of $\mathbf{g}$ to define the finite element approximation. In this way the a priori error analysis is separated in two parts: the error due to the regularization and that due to the discretization. We will analyze the first error in general, assuming a given approximation of $\mathbf{g}$ and considering afterwards some particular regularizations that can be used in practice.

For piecewise smooth boundary data, as in the case of the lid-driven cavity problem, it is natural to use as an approximation to $\mathrm{g}$ its Lagrange interpolation at continuity points with some appropriate definition at the discontinuities. This is a particular regularization and so we can apply our theory. We will show that this procedure produces an optimal order approximation for the lid-driven cavity problem improving, in particular, the result obtained in [9] where the order was suboptimal. Let us remark that, since in this example the solution belongs to $H^{s}(\Omega)$ for all $s<1$ (see [3, 19]), the best expected order for the error in the $L^{2}$ norm using quasi-uniform meshes is $O(h)$.

In the second part of the paper we introduce and analyze an a posteriori error estimator of the residual type. We will prove that the estimator is equivalent to appropriate norms of the error. Numerical examples will show that an adaptive procedure based on our estimator produce optimal order error estimates for the lid-driven cavity problem. 
Since (1.1) with $\mathbf{g}=0$ has been already analyzed, we restrict ourselves to study the case $\mathbf{f}=0$ and $\eta=0$, that is,

Problem 1.1. Given $\mathbf{g} \in \mathbf{L}^{2}(\Gamma)$ with

$$
\int_{\Gamma} \mathbf{g} \cdot \mathbf{n}=0
$$

find $(\mathbf{u}, p)$ such that

$$
\begin{aligned}
-\Delta \mathbf{u}+\nabla p=0 & & \text { in } \Omega \\
\operatorname{div} \mathbf{u}=0 & & \text { in } \Omega \\
\mathbf{u}=\mathbf{g} & & \text { on } \Gamma .
\end{aligned}
$$

The existence and uniqueness of solution is known. Indeed, we have

Proposition 1.1. Let $\Omega$ be a Lipschitz convex polygon or polyhedron, and $\mathbf{g} \in$ $\mathbf{L}^{2}(\Gamma)$ satisfying the compatibility condition (1.3). Then the Stokes system (1.4) has a unique solution $(\mathbf{u}, p) \in \mathbf{L}^{2}(\Omega) \times H^{-1}(\Omega) / \mathbb{R}$.

Moreover, there exists a constant $C$, depending only on $\Omega$, such that

$$
\|\mathbf{u}\|_{0, \Omega}+\|p\|_{H^{-1}(\Omega) / \mathbb{R}} \leq C\|\mathbf{g}\|_{0, \Gamma} .
$$

Proof. The existence of solution is proved in 16 in the two dimensional case and in 14 in the three dimensional case. Actually, in [16] the a priori estimate is proved only for smooth solutions but a standard density argument, as the one we use below in Proposition 2.2 can be applied to obtain the general case.

On the other hand, in 14 it is not explicitly stated that $p \in H^{-1}(\Omega)$. However, since $\mathbf{u} \in \mathbf{L}^{2}(\Omega)$ it follows immediately that $\nabla p \in H^{-2}(\Omega)$ from which one can get $p \in H^{-1}(\Omega)$ and (1.5) (see [16, page 317] and references therein). Let us also mention that the method used in 14 could also be applied in the two dimensional case as it was done for the case of the Laplace equation in [22].

The rest of the paper is organized as follows. In Section 2 we introduce the finite element approximation which is based in replacing the boundary datum $\mathbf{g}$ by smooth approximations $\mathbf{g}_{h}$. Then we develop the a priori error analysis which is divided in two subsections. In the first one we estimate the error between the exact solution of the original problem and the regularized one in terms of $\mathbf{g}-\mathbf{g}_{h}$. In the second subsection, considering some appropriate computable approximations, we analyze the error due to the finite element approximation of the regularized problem and prove a theorem which gives a bound for the total error in terms of fractional order norms of $\mathbf{g}$. Then, in Section 3 we consider the case of piecewise smooth data approximated by a suitable modification of the Lagrange interpolation. Section 4 deals with a posteriori error estimates. We introduce and analyze an error indicator for the regularized problem. Finally, in Section 5 , we present some numerical examples for the lid-driven cavity problem using two well known stable methods: the so called Mini element and the Hood-Taylor one.

\section{Finite element approximation and a priori estimates}

In this section we introduce the finite element approximation to Problem 1.1 and prove a priori error estimates. As we have mentioned, in general the solution $\mathbf{u}$ of this problem is not in $\mathbf{H}^{1}(\Omega)$ and so the standard finite element formulation and analysis cannot be applied. Therefore, to define the numerical approximation, 
we first approximate the original problem by more regular ones and then solve these problems by standard finite elements. Consequently, our error analysis is divided in two parts that we present in the following subsections. In the first one we analyze the error due to the regularization, while in the second one the finite element discretization error.

Given $\mathbf{g} \in \mathbf{L}^{2}(\Gamma)$, let $\mathbf{g}_{h} \in \mathbf{H}^{\frac{1}{2}}(\Gamma)$ be approximations of $\mathbf{g}$ such that

$$
\int_{\Gamma} \mathbf{g}_{h} \cdot \mathbf{n}=0
$$

and

$$
\lim _{h \rightarrow 0}\left\|\mathbf{g}-\mathbf{g}_{h}\right\|_{0, \Gamma}=0 .
$$

Here $h>0$ is an abstract parameter which afterwords will be related to the finite element meshes. The existence of approximations satisfying the compatibility condition (2.1) is not difficult to prove. Anyway we will construct explicit approximations later on using suitable interpolations or projections.

For each $h$, we consider the following regularized problem: find $\mathbf{u}(h)$ and $p(h)$, such that

$$
\begin{aligned}
-\Delta \mathbf{u}(h)+\nabla p(h) & =0 & & \text { in } \Omega \\
\operatorname{div} \mathbf{u}(h) & =0 & & \text { in } \Omega \\
\mathbf{u}(h) & =\mathbf{g}_{h} & & \text { on } \Gamma .
\end{aligned}
$$

This problem has a unique solution which, in view of (1.2), satisfies

$$
\|\mathbf{u}(h)\|_{1, \Omega}+\|p(h)\|_{L^{2}(\Omega) / \mathbb{R}} \leq C\left\|\mathbf{g}_{h}\right\|_{1 / 2, \Gamma} .
$$

The standard variational formulation of this regularized problem reads: find $\mathbf{u}(h) \in \mathbf{H}^{1}(\Omega)$ with $\mathbf{u}(h)=\mathbf{g}_{h}$ on $\Gamma$ and $p(h) \in L_{0}^{2}(\Omega)$ such that

$$
\begin{aligned}
(\nabla \mathbf{u}(h), \nabla \mathbf{v})-(\operatorname{div} \mathbf{v}, p(h))=0 & \forall \mathbf{v} \in \mathbf{H}_{0}^{1}(\Omega) \\
(\operatorname{div} \mathbf{u}(h), q)=0 & \forall q \in L_{0}^{2}(\Omega) .
\end{aligned}
$$

2.1. Analysis of the error due to the approximation of the boundary datum. We will make use of the following well known result.

Proposition 2.1. Let $\Omega$ be a convex Lipschitz polygonal or polyhedral domain and $\mathbf{f} \in \mathbf{L}^{2}(\Omega)$. Then the system

$$
\begin{aligned}
-\Delta \phi+\nabla q & =\mathbf{f} & & \text { in } \Omega \\
\operatorname{div} \phi & =0 & & \text { in } \Omega \\
\phi & =0 & & \text { on } \Gamma .
\end{aligned}
$$

has a unique solution $(\phi, q) \in \mathbf{H}^{2}(\Omega) \cap \mathbf{H}_{0}^{1}(\Omega) \times H^{1}(\Omega) / \mathbb{R}$ which satisfies the following a priori estimate

$$
\|\phi\|_{2, \Omega}+\|q\|_{H^{1}(\Omega) / \mathbb{R}} \leq C\|\mathbf{f}\|_{0, \Omega} .
$$

Proof. This is proved in [17. Theorem 2] for $d=2$ and in [13. Theorem 9.20 (b)] for $d=3$.

The result given in the next lemma is known but we outline the proof in order to make explicit the dependence of the involved constant on $s$. We will denote by $\Gamma_{i}, 1 \leq i \leq N_{e}$, the edges or faces of $\Gamma$. 
Lemma 2.1. There exists a constant $C$ independent of $s$ such that, for $0 \leq s<\frac{1}{2}$,

$$
\|f\|_{-s, \Gamma_{i}} \leq \frac{C}{1-2 s}\|f\|_{-s, \Gamma}, \quad \forall f \in L^{2}(\Gamma) .
$$

Proof. Given $\phi \in H^{s}\left(\Gamma_{i}\right)$ let $\widetilde{\phi}$ be its extension by 0 to $\Gamma$. Tracing constants in the proof of [18, Th. 11.4 in Chapt. 1], we can show that for $0 \leq s<\frac{1}{2}$,

$$
\|\widetilde{\phi}\|_{s, \Gamma} \leq \frac{C}{1-2 s}\|\phi\|_{s, \Gamma_{i}} \quad \forall \phi \in H^{s}\left(\Gamma_{i}\right),
$$

and then, we have

$$
\begin{aligned}
\|f\|_{-s, \Gamma_{i}} & =\sup _{0 \neq \phi \in H^{s}\left(\Gamma_{i}\right)} \frac{\int_{\Gamma_{i}} f \phi d s}{\|\phi\|_{s, \Gamma_{i}}} \\
& =\sup _{0 \neq \phi \in H^{s}\left(\Gamma_{i}\right)} \frac{\int_{\Gamma_{i}} f \widetilde{\phi} d s}{\|\widetilde{\phi}\|_{s, \Gamma}} \frac{\|\widetilde{\phi}\|_{s, \Gamma}}{\|\phi\|_{s, \Gamma_{i}}}
\end{aligned}
$$

which yields

$$
\begin{aligned}
\|f\|_{-s, \Gamma_{i}} & \leq \frac{C}{1-2 s} \sup _{0 \neq \phi \in H^{s}\left(\Gamma_{i}\right)} \frac{\int_{\Gamma_{i}} f \widetilde{\phi} d s}{\|\widetilde{\phi}\|_{s, \Gamma}} \\
& \leq \frac{C}{1-2 s} \sup _{0 \neq \phi \in H^{s}(\Gamma)} \frac{\int_{\Gamma_{i}} f \phi d s}{\|\phi\|_{s, \Gamma}}
\end{aligned}
$$

that is (2.7).

In the following proposition we estimate the error between the solutions $(\mathbf{u}, p)$ of (1.4) and $(\mathbf{u}(h), p(h))$ of (2.3) in the $L^{2}(\Omega)$-norm for the velocity and in $H^{-1}(\Omega) / \mathbb{R}$ norm for the pressure.

Proposition 2.2. Let $\Omega$ be a convex Lipschitz polygonal or polyhedral domain and $(\mathbf{u}, p)$ and $(\mathbf{u}(h), p(h))$ be the solutions of (1.4) and (2.3), respectively. Then, there exists a constants $C$, independent of $h$, such that for $0 \leq s<\frac{1}{2}$,

$$
\|\mathbf{u}-\mathbf{u}(h)\|_{0, \Omega}+\|p-p(h)\|_{H^{-1}(\Omega) / \mathbb{R}} \leq \frac{C}{1-2 s}\left\|\mathbf{g}-\mathbf{g}_{h}\right\|_{-s, \Gamma} .
$$

Proof. First we will estimate the $L^{2}(\Omega)$-norm of $\mathbf{v}:=\mathbf{u}-\mathbf{u}(h)$. Since $\Omega$ is convex, we know from Proposition [2.1, that there exist $\phi \in \mathbf{H}^{2}(\Omega) \cap \mathbf{H}_{0}^{1}(\Omega)$ and $q \in$ $H^{1}(\Omega) \cap L_{0}^{2}(\Omega)$ solutions of the following system,

$$
\begin{aligned}
-\Delta \phi+\nabla q & =\mathbf{v} & & \text { in } \Omega \\
\operatorname{div} \phi & =0 & & \text { in } \Omega \\
\phi & =0 & & \text { on } \Gamma .
\end{aligned}
$$

Take $h_{1}$ another value of the parameter. Then, taking into account (2.5), we have

$$
\begin{aligned}
\left(\mathbf{u}\left(h_{1}\right)-\right. & \mathbf{u}(h), \mathbf{v})_{\Omega}=\left(\mathbf{u}\left(h_{1}\right)-\mathbf{u}(h),-\Delta \boldsymbol{\phi}+\nabla q\right)_{\Omega} \\
= & \left(\nabla\left(\mathbf{u}\left(h_{1}\right)-\mathbf{u}(h)\right), \nabla \phi\right)_{\Omega}-\left(\mathbf{u}\left(h_{1}\right)-\mathbf{u}(h), \frac{\partial \phi}{\partial \mathbf{n}}\right)_{\Gamma} \\
& -\left(\operatorname{div}\left(\mathbf{u}\left(h_{1}\right)-\mathbf{u}(h)\right), q\right)_{\Omega}+\left(\left(\mathbf{u}\left(h_{1}\right)-\mathbf{u}(h)\right) \cdot \mathbf{n}, q\right)_{\Gamma} \\
= & -\left(\mathbf{g}_{h_{1}}-\mathbf{g}_{h}, \frac{\partial \phi}{\partial \mathbf{n}}\right)_{\Gamma}+\left(\left(\mathbf{g}_{h_{1}}-\mathbf{g}_{h}\right) \cdot \mathbf{n}, q\right)_{\Gamma} .
\end{aligned}
$$


Summarizing,

$$
\left(\mathbf{u}\left(h_{1}\right)-\mathbf{u}(h), \mathbf{v}\right)_{\Omega}=-\left(\mathbf{g}_{h_{1}}-\mathbf{g}_{h}, \frac{\partial \phi}{\partial \mathbf{n}}\right)_{\Gamma}+\left(\left(\mathbf{g}_{h_{1}}-\mathbf{g}_{h}\right) \cdot \mathbf{n}, q\right)_{\Gamma} .
$$

Since, from (1.5) and (2.2) we know that, for $h_{1} \rightarrow 0$,

$$
\left\|\mathbf{u}-\mathbf{u}\left(h_{1}\right)\right\|_{0, \Omega} \leq C\left\|\mathbf{g}-\mathbf{g}_{h_{1}}\right\|_{0, \Gamma} \rightarrow 0,
$$

taking $h_{1} \rightarrow 0$ in (2.10), we obtain

$$
(\mathbf{u}-\mathbf{u}(h), \mathbf{v})_{\Omega}=-\left(\mathbf{g}-\mathbf{g}_{h}, \frac{\partial \phi}{\partial \mathbf{n}}\right)_{\Gamma}+\left(\left(\mathbf{g}-\mathbf{g}_{h}\right) \cdot \mathbf{n}, q\right)_{\Gamma} .
$$

We estimate the right hand side in terms of $\left\|\mathbf{g}-\mathbf{g}_{h}\right\|_{H^{-s}(\Gamma)}$. For the second term we note that while $q \in H^{\frac{1}{2}}(\Gamma)$, due to the discontinuities of $\mathbf{n}$ we can not assure that $q \mathbf{n} \in H^{\frac{1}{2}}(\Gamma)$. Therefore, with $0 \leq s<\frac{1}{2}$, we have

$$
\begin{aligned}
\left(\mathbf{g}-\mathbf{g}_{h}, q \mathbf{n}\right)_{\Gamma} & =\sum_{i=1}^{N_{e}}\left(\mathbf{g}-\mathbf{g}_{h}, q \mathbf{n}\right)_{\Gamma_{i}} \leq \sum_{i=1}^{N_{e}}\left\|\mathbf{g}-\mathbf{g}_{h}\right\|_{-s, \Gamma_{i}}\|q \mathbf{n}\|_{s, \Gamma_{i}} \\
& \leq C\left(\sum_{i=1}^{N_{e}}\left\|\mathbf{g}-\mathbf{g}_{h}\right\|_{-s, \Gamma_{i}}^{2}\right)^{\frac{1}{2}}\|q\|_{1, \Omega} \leq \frac{C}{1-2 s}\left\|\mathbf{g}-\mathbf{g}_{h}\right\|_{-s, \Gamma}\|q\|_{1, \Omega}
\end{aligned}
$$

where, in the last inequality, we have used (2.7). With a similar argument, we obtain for the first term in the right-hand side of (2.11) the estimate

$$
\left(\mathbf{g}-\mathbf{g}_{h}, \frac{\partial \phi}{\partial \mathbf{n}}\right)_{\Gamma} \leq \frac{C}{1-2 s}\left\|\mathbf{g}-\mathbf{g}_{h}\right\|_{-s, \Gamma}\|\phi\|_{2, \Omega} .
$$

Hence, from (2.11) and the a priori estimate (2.6) we have

$$
\begin{aligned}
\|\mathbf{u}-\mathbf{u}(h)\|_{0, \Omega}^{2} & =(\mathbf{u}-\mathbf{u}(h), \mathbf{v}) \\
& \leq \frac{C}{1-2 s}\left\|\mathbf{g}-\mathbf{g}_{h}\right\|_{-s, \Gamma}\left(\|\boldsymbol{\phi}\|_{2, \Omega}+\|q\|_{1, \Omega}\right) \\
& \leq \frac{C}{1-2 s}\left\|\mathbf{g}-\mathbf{g}_{h}\right\|_{-s, \Gamma}\|\mathbf{u}-\mathbf{u}(h)\|_{0, \Omega}
\end{aligned}
$$

and so,

$$
\|\mathbf{u}-\mathbf{u}(h)\|_{0, \Omega} \leq \frac{C}{1-2 s}\left\|\mathbf{g}-\mathbf{g}_{h}\right\|_{-s, \Gamma} .
$$

Now, for the error in the pressure we have

$$
\begin{aligned}
\|p-p(h)\|_{H^{-1}(\Omega) / \mathbb{R}} & \leq C\|\nabla(p-p(h))\|_{-2, \Omega}=C\|\Delta(\mathbf{u}-\mathbf{u}(h))\|_{-2, \Omega} \\
& \leq C\|\mathbf{u}-\mathbf{u}(h)\|_{0, \Omega} \leq \frac{C}{1-2 s}\left\|\mathbf{g}-\mathbf{g}_{h}\right\|_{-s, \Gamma}
\end{aligned}
$$

which concludes the proof.

Remark 2.1. The estimate of the previous proposition can be compared with 4, Lemma 2.12] where the corresponding result for the approximation of a Poisson equation with a non smooth Dirichlet boundary datum is considered. A constant independent of $s$ is obtained in [4, while our estimate contains a factor $C /(1-2 s)$. Indeed, we could bound the first term in the right-hand side of (2.11) exactly as in 4. However, the slightly worse factor $C /(1-2 s)$ arises due to the presence of the second term which involves the pressure $q$. 
2.2. Analysis of the finite element approximation error. Let $\left\{\mathcal{T}_{h}\right\}, h>0$, be a family of meshes of $\Omega$, which is assumed to be shape-regular, with $h$ being the maximum diameter of the elements in $\mathcal{T}_{h}$. Each mesh $\mathcal{T}_{h}$ induces a mesh $\mathcal{T}_{\Gamma, h}$ along the boundary fitted with the edges/faces $\Gamma_{i}, i=1, \ldots, N_{e}$.

We consider a family of pairs $\mathbf{V}_{h}=\mathbf{W}_{h} \cap \mathbf{H}_{0}^{1}(\Omega)$ and $Q_{h} \subset L_{0}^{2}(\Omega)$ of finite element spaces, with $\mathbf{W}_{h} \subset \mathbf{H}^{1}(\Omega)$, which are uniformly stable for the Stokes problem, that is, the following inf-sup condition is satisfied for some $\beta>0$ independent of $h$ (see, e.g., [6, Chap.8])

$$
\sup _{\mathbf{v}_{h} \in \mathbf{V}_{h}} \frac{\left(q_{h}, \operatorname{div} \mathbf{v}_{h}\right)_{0, \Omega}}{\left\|\mathbf{v}_{h}\right\|_{1, \Omega}} \geq \beta\left\|q_{h}\right\|_{0, \Omega}, \quad \forall q_{h} \in Q_{h}, \quad \forall h>0 .
$$

Moreover, we assume that

$$
\left[\mathcal{P}_{1}\left(\mathcal{T}_{h}\right) \cap H^{1}(\Omega)\right]^{d} \subseteq \mathbf{W}_{h}, \quad \mathcal{P}_{0}\left(\mathcal{T}_{h}\right) \subseteq Q_{h},
$$

where $\mathcal{P}_{k}\left(\mathcal{T}_{h}\right)$ stands for the vector space of piecewise polynomials of degree not grater than $k$ on the mesh $\mathcal{T}_{h}$. In the following we shall use interpolant operators onto the discrete spaces $\mathbf{W}_{h}$ and $Q_{h}$. For functions $\phi \in \mathbf{H}^{2}(\Omega)$, we define $\phi^{I} \in \mathbf{W}_{h}$ as the continuous piecewise linear Lagrange interpolation of $\phi$. The following error estimates are well known:

$$
\left\|\phi-\phi^{I}\right\|_{m, T} \leq C h^{2-m}|\phi|_{2, T}, \quad m=0,1 \quad \text { for all } \phi \in \mathbf{H}^{2}(\Omega) .
$$

Let $P_{0}$ be the $L^{2}$-projection of $L_{0}^{2}(\Omega)$ onto $\mathcal{P}^{0}\left(\mathcal{T}_{h}\right)$, it is well known that

$$
\left\|q-P_{0} q\right\|_{0, T} \leq C h|q|_{1, \Omega} \text { for all } q \in H^{1}(\Omega) .
$$

From now on, we assume that $\mathbf{g}_{h}$ is the trace of a function $E \mathbf{g}_{h} \in \mathbf{W}_{h}$, for example, it is enough to assume that $\mathbf{g}_{h}$ is continuous and piecewise linear. Moreover, it is known that $E \mathbf{g}_{h}$ can be chosen such that $\left\|E \mathbf{g}_{h}\right\|_{1, \Omega} \leq C\left\|\mathbf{g}_{h}\right\|_{\frac{1}{2}, \Gamma}$.

We consider the finite element approximation of (2.5) that reads: find $\mathbf{u}_{h} \in \mathbf{W}_{h}$ and $p_{h} \in Q_{h}$ such that $\mathbf{u}_{h}=\mathbf{g}_{h}$ on $\Gamma$ and

$$
\begin{aligned}
\left(\nabla \mathbf{u}_{h}, \nabla \mathbf{v}_{h}\right)-\left(\operatorname{div} \mathbf{v}_{h}, p_{h}\right) & =0 & & \forall \mathbf{v}_{h} \in \mathbf{V}_{h} \\
\left(\operatorname{div} \mathbf{u}_{h}, q_{h}\right) & =0 & & \forall q_{h} \in Q_{h} .
\end{aligned}
$$

By taking $\mathbf{v}_{h}=\mathbf{u}_{h}-E \mathbf{g}_{h}$ and $q_{h}=p_{h}$ in (2.15), and using the inf-sup condition, we obtain existence and uniqueness and the estimate

$$
\left\|\mathbf{u}_{h}\right\|_{1, \Omega}+\left\|p_{h}\right\|_{0, \Omega} \leq C\left\|\mathbf{g}_{h}\right\|_{\frac{1}{2}, \Gamma} .
$$

In the following proposition we estimate the finite element error in norms corresponding with the ones used in Proposition 2.2.

Proposition 2.3. Let $(\mathbf{u}(h), p(h)) \in \mathbf{H}^{1}(\Omega) \times L_{0}^{2}(\Omega)$ with $\mathbf{u}(h)=\mathbf{g}_{h}$ on $\Gamma$ and $\left(\mathbf{u}_{h}, p_{h}\right) \in \mathbf{W}_{h} \times Q_{h}$ with $\mathbf{u}_{h}=E \mathbf{g}_{h}+\mathbf{u}_{0 h}$ be the solutions of (2.5) and (2.15), respectively. Then we have

$$
\left\|\mathbf{u}(h)-\mathbf{u}_{h}\right\|_{0, \Omega}+\left\|p(h)-p_{h}\right\|_{H^{-1}(\Omega) / \mathbb{R}} \leq C h\left\|\mathbf{g}_{h}\right\|_{\frac{1}{2}, \Gamma} .
$$

Proof. Subtracting (2.15) from (2.5), we get the following error equations:

$$
\begin{aligned}
\left(\nabla\left(\mathbf{u}(h)-\mathbf{u}_{h}\right), \nabla \mathbf{v}_{h}\right)-\left(\operatorname{div} \mathbf{v}_{h}, p(h)-p_{h}\right) & =0 & & \forall \mathbf{v}_{h} \in V_{h} \\
\left(\operatorname{div}\left(\mathbf{u}(h)-\mathbf{u}_{h}\right), q_{h}\right) & =0 & & \forall q_{h} \in Q_{h} .
\end{aligned}
$$


In order to use a duality argument, we introduce the following system: find $(\phi, q)$ satisfying

$$
\begin{aligned}
-\Delta \phi+\nabla q & =\mathbf{u}(h)-\mathbf{u}_{h} & & \text { in } \Omega \\
\operatorname{div} \phi & =0 & & \text { in } \Omega \\
\phi & =0 & & \text { on } \Gamma .
\end{aligned}
$$

From Proposition 2.1, $\phi \in \mathbf{H}^{2}(\Omega) \cap \mathbf{H}_{0}^{1}(\Omega)$ and $q \in H^{1}(\Omega) \cap L_{0}^{2}(\Omega)$ with the a priori estimate (2.6). We have

$$
\left\|\mathbf{u}(h)-\mathbf{u}_{h}\right\|_{0, \Omega}^{2}=\left(\mathbf{u}(h)-\mathbf{u}_{h},-\Delta \phi+\nabla q\right)
$$

then integration by parts, the error equations (2.18), the approximation properties (2.14) and (2.16), the fact that $\mathbf{u}(h)=\mathbf{u}_{h}=\mathbf{g}_{h}$ on the boundary, and the a priori estimates (2.4) and (2.16), give

$$
\begin{aligned}
\| \mathbf{u}(h)- & \mathbf{u}_{h} \|_{0, \Omega}^{2} \\
= & \left(\nabla\left(\mathbf{u}(h)-\mathbf{u}_{h}\right), \nabla \phi\right)-\left(\operatorname{div}\left(\mathbf{u}(h)-\mathbf{u}_{h}\right), q\right)-\left(\operatorname{div} \phi, p(h)-p_{h}\right) \\
= & \left(\nabla\left(\mathbf{u}(h)-\mathbf{u}_{h}\right), \nabla\left(\phi-\phi^{I}\right)\right) \\
& \quad-\left(\operatorname{div}\left(\boldsymbol{\phi}-\boldsymbol{\phi}^{I}\right), p(h)-p_{h}\right)-\left(\operatorname{div}\left(\mathbf{u}(h)-\mathbf{u}_{h}\right), q-P_{0} q\right) \\
\leq & C h\left(|\phi|_{2, \Omega}+|q|_{1, \Omega}\right)\left\|\nabla\left(\mathbf{u}(h)-\mathbf{u}_{h}\right)\right\|_{0, \Omega}+C h|\phi|_{2, \Omega}\left\|p(h)-p_{h}\right\|_{0, \Omega} \\
\leq & C h\left\|\mathbf{u}(h)-\mathbf{u}_{h}\right\|_{0, \Omega}\left(\|\nabla \mathbf{u}(h)\|_{0, \Omega}+\left\|\nabla \mathbf{u}_{h}\right\|_{0, \Omega}+\|p(h)\|_{0, \Omega}+\left\|p_{h}\right\|_{0, \Omega}\right) \\
\leq & C h\left\|\mathbf{u}(h)-\mathbf{u}_{h}\right\|_{0, \Omega}\left\|\mathbf{g}_{h}\right\|_{\frac{1}{2}, \Gamma}
\end{aligned}
$$

which provides the desired estimate for the velocity field

$$
\left\|\mathbf{u}(h)-\mathbf{u}_{h}\right\|_{0, \Omega} \leq C h\left\|\mathbf{g}_{h}\right\|_{\frac{1}{2}, \Gamma} .
$$

Let us now estimate $p(h)-p_{h}$. Since $p(h)-p_{h} \in L_{0}^{2}(\Omega)$, we have

$$
\left\|p(h)-p_{h}\right\|_{H^{-1}(\Omega) / \mathbb{R}}=\sup _{\substack{q \in H_{0}^{1}(\Omega) \\ \int_{\Omega} q=0}} \frac{\left(p(h)-p_{h}, q\right)}{\|q\|_{1, \Omega}} .
$$

Given $q \in H_{0}^{1}(\Omega)$ with $\int_{\Omega} q=0$, we know that there exists $\boldsymbol{\psi} \in \mathbf{H}_{0}^{2}(\Omega)$ such that 20. Theorem 1]

$$
\operatorname{div} \boldsymbol{\psi}=q \quad \text { in } \Omega, \quad\|\boldsymbol{\psi}\|_{2, \Omega} \leq C\|q\|_{1, \Omega} .
$$

Then using the interpolant $\psi^{I}$ as in (2.14), and the error equation (2.18), we have

$$
\begin{aligned}
\left(p(h)-p_{h}, q\right)= & \left(p(h)-p_{h}, \operatorname{div} \boldsymbol{\psi}\right) \\
= & \left(p(h)-p_{h}, \operatorname{div}\left(\boldsymbol{\psi}-\boldsymbol{\psi}^{I}\right)\right)+\left(\nabla\left(\mathbf{u}(h)-\mathbf{u}_{h}\right), \nabla \psi^{I}\right) \\
= & \left(p(h)-p_{h}, \operatorname{div}\left(\boldsymbol{\psi}-\boldsymbol{\psi}^{I}\right)\right)-\left(\nabla\left(\mathbf{u}(h)-\mathbf{u}_{h}\right), \nabla\left(\boldsymbol{\psi}-\boldsymbol{\psi}^{I}\right)\right) \\
& \quad+\left(\nabla\left(\mathbf{u}(h)-\mathbf{u}_{h}\right), \nabla \boldsymbol{\psi}\right) .
\end{aligned}
$$

Integrating by parts the last term, we have

$$
\begin{aligned}
\left(p(h)-p_{h}, q\right)= & \left(p(h)-p_{h}, \operatorname{div}\left(\boldsymbol{\psi}-\boldsymbol{\psi}^{I}\right)\right)-\left(\nabla\left(\mathbf{u}(h)-\mathbf{u}_{h}\right), \nabla\left(\boldsymbol{\psi}-\boldsymbol{\psi}^{I}\right)\right) \\
& -\left(\mathbf{u}(h)-\mathbf{u}_{h}, \Delta \boldsymbol{\psi}\right) .
\end{aligned}
$$


Then we obtain

$$
\begin{array}{rl}
\left(p(h)-p_{h}, q\right) \leq C & h|\boldsymbol{\psi}|_{2, \Omega}\left(\|p(h)\|_{0, \Omega}+\left\|p_{h}\right\|_{0, \Omega}\right) \\
& +C h\left(\|\nabla \mathbf{u}(h)\|_{0, \Omega}+\left\|\nabla \mathbf{u}_{h}\right\|_{0, \Omega}\right)|\boldsymbol{\psi}|_{2, \Omega} \\
& +\left\|\mathbf{u}(h)-\mathbf{u}_{h}\right\|_{0, \Omega}|\boldsymbol{\psi}|_{2, \Omega} \\
\leq C & {\left[h\left(\|p(h)\|_{0, \Omega}+\left\|p_{h}\right\|_{0, \Omega}+\|\nabla \mathbf{u}(h)\|_{0, \Omega}+\left\|\nabla \mathbf{u}_{h}\right\|_{0, \Omega}\right)\right.} \\
& \left.+\left\|\mathbf{u}(h)-\mathbf{u}_{h}\right\|_{0, \Omega}\right]\|q\|_{1, \Omega} .
\end{array}
$$

Substituting this inequality in (2.21) implies

$$
\begin{aligned}
\left\|p(h)-p_{h}\right\|_{H^{-1}(\Omega) / \mathbb{R}} & \leq C h\left(\|p(h)\|_{0, \Omega}+\left\|p_{h}\right\|_{0, \Omega}+\|\nabla \mathbf{u}(h)\|_{0, \Omega}+\left\|\nabla \mathbf{u}_{h}\right\|_{0, \Omega}\right) \\
& +\left\|\mathbf{u}(h)-\mathbf{u}_{h}\right\|_{0, \Omega} .
\end{aligned}
$$

Then the stability estimates (2.4) and (2.16) joint with (2.20), give

$$
\left\|p(h)-p_{h}\right\|_{H^{-1}(\Omega) / \mathbb{R}} \leq C h\left\|\mathbf{g}_{h}\right\|_{\frac{1}{2}, \Gamma}
$$

that together with (2.20) concludes the proof.

The regularization of the boundary datum $\mathrm{g}$ could be obtained by finite element discretization. By construction of the mesh $\mathcal{T}_{h}$, the boundary $\Gamma$ is subdivided into boundary elements fitted with the edges/faces $\Gamma_{i}, i=1, \ldots, N_{e}$ and $\mathcal{T}_{\Gamma, h}$ denotes the mesh along the boundary. Let $h_{\Gamma}$ be the maximum diameter of the elements in $\mathcal{T}_{\Gamma, h}$ and define the discrete space on the boundary as

$$
\mathbf{G}_{h}=\left\{\mathbf{z}_{h} \in \mathbf{C}^{0}(\Gamma): \mathbf{z}_{h} \in \mathcal{P}^{1}(E) \forall E \in \mathcal{T}_{\Gamma, h}\right\} .
$$

Then the function $\mathbf{g}_{h}$ can be obtained either as the $\mathbf{L}^{2}(\Gamma)$-projection of $\mathbf{g}$ onto the space $\mathbf{G}_{h}$, or using the Carstensen interpolant $\mathbf{C}_{h} \mathbf{g}$ of $\mathbf{g}$, see [10, or by a suitable Lagrange interpolation, see Section 3 It is straightforward to check that both the $L^{2}$-projection and the Carstensen interpolant provide approximations $\mathbf{g}_{h}$ of $\mathbf{g}$ which satisfy the compatibility condition (2.1), while this is not always the case for the standard Lagrange interpolation. Moreover we can show the following regularization error estimates for $\mathbf{g}_{h}$ (see [4, Lemmata 2.13 and A.2]):

Proposition 2.4. Let $\mathbf{g}_{h} \in \mathbf{G}_{h}$ be either the piecewise linear Carstensen interpolant of $\mathbf{g}$ or the $L^{2}(\Gamma)$-projection on the continuous piecewise linear functions, then we have

$$
\left\|\mathbf{g}-\mathbf{g}_{h}\right\|_{-s, \Gamma} \leq C h^{s+t}\|\mathbf{g}\|_{t, \Gamma}, \quad \forall \mathbf{g} \in H^{t}(\Gamma), s, t \in[0,1] .
$$

We also have

$$
\left\|\mathbf{g}_{h}\right\|_{t, \Gamma} \leq C\|\mathbf{g}\|_{t, \Gamma} \quad \forall \mathbf{g} \in H^{t}(\Gamma), t \in[0,1],
$$

where, for $t>0$, it is assumed that the mesh $\mathcal{T}_{\Gamma, h}$ is quasi-uniform.

Proof. Inequality (2.26) is proved in [4, Lemma A.2] for $\mathbf{g}_{h}$ being the Carstensen interpolant, and in [4, Remark A.3] when $\mathbf{g}_{h}$ is the $L^{2}$-projection on piecewise linear functions on $\Gamma$.

Inequality (2.27) for $t=0$ is also proved in 4. For $t>0$ we can proceed as follows.

Let $\Pi_{h}: \mathbf{H}^{1}(\Gamma) \rightarrow \mathbf{G}_{h}$ be the Clément's operator such that for all $\mathbf{g} \in \mathbf{H}^{1}(\Gamma)$

$$
\left\|\mathbf{g}-\Pi_{h} \mathbf{g}\right\|_{r, \Gamma} \leq c\left(\sum_{K} h_{K}^{2-2 r}\|\mathbf{g}\|_{1, K}^{2}\right)^{\frac{1}{2}} \quad \text { for } r=0,1 .
$$


Then, if the mesh is quasi-uniform we can use an inverse inequality and obtain

$$
\begin{aligned}
\left\|\nabla \mathbf{g}_{h}\right\|_{0, \Gamma} & \leq\left\|\nabla \Pi_{h} \mathbf{g}\right\|_{0, \Gamma}+\left\|\nabla\left(\mathbf{g}_{h}-\Pi_{h} \mathbf{g}\right)\right\|_{0, \Gamma} \\
& \leq c\|\mathbf{g}\|_{1, \Gamma}+\frac{C_{I}}{h}\left\|\mathbf{g}_{h}-\Pi_{h} \mathbf{g}\right\|_{0, \Gamma} \\
& \leq c\|\mathbf{g}\|_{1, \Gamma}+\frac{C_{I}}{h}\left\|\mathbf{g}_{h}-\mathbf{g}\right\|_{0, \Gamma}+\frac{C_{I}}{h}\left\|\mathbf{g}-\Pi_{h} \mathbf{g}\right\|_{0, \Gamma} \leq C\|\mathbf{g}\|_{1, \Gamma} .
\end{aligned}
$$

Then by interpolation of Sobolev spaces (see, e.g., [8, Prop. 14.1.5]) we get (2.27).

The bounds (2.9) and (2.17) together with the inequalities in Proposition 2.4 give the following result.

Theorem 2.1. Let $\Omega$ be a convex polygonal or polyhedral domain. If the family of meshes $\mathcal{T}_{\Gamma, h}$ is quasi-uniform and $\mathbf{g}_{h}$ is given as in Proposition 2.4 then, for $0 \leq t<\frac{1}{2}$ and $\mathbf{g} \in H^{t}(\Gamma)$, we have

$$
\left\|\mathbf{u}-\mathbf{u}_{h}\right\|_{0, \Omega}+\left\|p-p_{h}\right\|_{H^{-1}(\Omega) / \mathbb{R}} \leq C|\log h| h^{\frac{1}{2}+t}\|\mathbf{g}\|_{t, \Gamma} .
$$

Proof. From Proposition 2.2 and (2.26), we have for $0 \leq s<\frac{1}{2}$

$$
\|\mathbf{u}-\mathbf{u}(h)\|_{0, \Omega}+\|p-p(h)\|_{H^{-1}(\Omega) / \mathbb{R}} \leq \frac{C}{1-2 s} h^{s+t}\|\mathbf{g}\|_{t, \Gamma} .
$$

Taking $s=1 / 2+1 / \log h<1 / 2$ we obtain

$$
\|\mathbf{u}-\mathbf{u}(h)\|_{0, \Omega}+\|p-p(h)\|_{H^{-1}(\Omega) / \mathbb{R}} \leq C h^{\frac{1}{2}+t}|\log h|\|\mathbf{g}\|_{t, \Gamma} .
$$

On the other hand, from Proposition 2.3 ,

$$
\left\|\mathbf{u}(h)-\mathbf{u}_{h}\right\|_{0, \Omega}+\left\|p(h)-p_{h}\right\|_{H^{-1}(\Omega) / \mathbb{R}} \leq C h\left\|\mathbf{g}_{h}\right\|_{\frac{1}{2}, \Gamma} .
$$

Now, using an inverse inequality ([12, Theorem 4.1]) and (2.27), we obtain

$$
\left\|\mathbf{g}_{h}\right\|_{\frac{1}{2}, \Gamma} \leq C h^{t-\frac{1}{2}}\left\|\mathbf{g}_{h}\right\|_{t, \Gamma} \leq C h^{t-\frac{1}{2}}\|\mathbf{g}\|_{t, \Gamma},
$$

which substituted in (2.30) gives,

$$
\left\|\mathbf{u}(h)-\mathbf{u}_{h}\right\|_{0, \Omega}+\left\|p(h)-p_{h}\right\|_{H^{-1}(\Omega)} \leq C h^{\frac{1}{2}+t}\|\mathbf{g}\|_{t, \Gamma} .
$$

Combining (2.29) and (2.31) we arrive at the desired estimate (2.28).

\section{A PRIORI ERROR ESTIMATES FOR PIECEWISE SMOOTH BOUNDARY DATA}

In this section we analyze the approximation of piecewise smooth data, in particular, our results can be applied to the lid-driven cavity problem. In practice, the most usual way to deal with the non-homogeneous Dirichlet boundary condition is to use the Lagrange interpolation or a simple modification of it, to treat discontinuities and to obtain a compatible approximation $\mathbf{g}_{h}$.

We shall use the following notation for the norm of $\mathbf{g}$

$$
\|\mathbf{g}\|_{k, \Gamma}=\left(\sum_{i=1}^{N_{e}}\|\mathbf{g}\|_{k, \Gamma_{i}}^{2}\right)^{\frac{1}{2}} .
$$

In the following, we consider separately the case $d=2$ or $d=3$. 
3.1. Two dimensional case. Let $\mathbf{g}=\left(g_{1}, g_{2}\right): \Gamma \rightarrow \mathbb{R}^{2}$ be such that $\left.\mathbf{g}\right|_{\Gamma_{i}} \in$ $\mathbf{H}^{1}\left(\Gamma_{i}\right)$ for $i=1, \ldots, N_{e}$, where $\Gamma_{i}$ are the boundary segments $\Gamma_{i}=\left[A_{i}, A_{i+1}\right]$ (with $A_{N_{e}+1}=A_{1}$ ) and $A_{i}, i=1, \ldots, N_{e}$ are the boundary vertices. We observe that $\mathbf{g} \in \mathbf{H}^{s}(\Gamma)$ with $0 \leq s<\frac{1}{2}$. Indeed, let us set $\mathbf{g}_{i}=\left.\mathbf{g}\right|_{\Gamma_{i}}$. Since, for $0 \leq s<\frac{1}{2}$, $H^{1}\left(\Gamma_{i}\right) \subset H^{s}\left(\Gamma_{i}\right)$, we have that the extension by zero $\tilde{\mathbf{g}}_{i}$ of $\mathbf{g}_{i} \in \mathbf{H}^{s}\left(\Gamma_{i}\right)$ belongs to $\mathbf{H}^{s}(\Gamma)$ (see [18, Th.11.4 in Chapt.1]) and, thanks to (2.8),

$$
\left\|\tilde{\mathbf{g}}_{i}\right\|_{s, \Gamma} \leq \frac{C}{1-2 s}\left\|\mathbf{g}_{i}\right\|_{s, \Gamma_{i}} .
$$

Then $\mathbf{g}=\sum_{i=1}^{N_{e}} \tilde{\mathbf{g}}_{i}$ belongs to $\mathbf{H}^{s}(\Gamma)$, with

$$
\|\mathbf{g}\|_{s, \Gamma} \leq \frac{C}{1-2 s}\|\mathbf{g}\|_{1, \Gamma} .
$$

We denote by $B_{i}, 1 \leq i \leq M$, the boundary nodes of the mesh numbered consecutively and set $B_{M+1}=B_{1}$ (of course these nodes depend on $h$ but we omit this in the notation for simplicity) and $h_{i}=\left|B_{i+1}-B_{i}\right|$. In principle, we would define $\mathbf{g}_{h}$ as the continuous piecewise linear vector field on $\Gamma$ such that $\mathbf{g}_{h}\left(B_{j}\right)=\mathbf{g}\left(B_{j}\right)$ if $\mathbf{g}$ is continuous in $B_{j}$ and $\mathbf{g}_{h}\left(B_{j}\right)=\mathbf{g}\left(B_{j}^{-}\right)$or $\mathbf{g}_{h}\left(B_{j}\right)=\mathbf{g}\left(B_{j}^{+}\right)$, or some average of these two values, if not. Notice that $\left|\mathbf{g}_{h}\left(B_{j}\right)\right| \leq\|\mathbf{g}\|_{L^{\infty}(\Gamma)}$. However, in general, this definition does not satisfy the compatibility condition (2.1). We now show how to enforce compatibility by a simple modification.

Lemma 3.1. Given $\mathbf{g} \in \mathbf{L}^{2}(\Gamma)$ such that $\left.\mathbf{g}\right|_{\Gamma_{i}} \in \mathbf{H}^{1}\left(\Gamma_{i}\right)$ for $i=1, \ldots, N_{e}$, there exists a piecewise linear function $\mathbf{g}_{h}$ which is a modified Lagrange interpolant of $\mathbf{g}$ satisfying the compatibility condition (2.1). Moreover,

$$
\left\|\mathbf{g}_{h}\right\|_{L^{\infty}(\Gamma)} \leq C\|\mathbf{g}\|_{1, \Gamma} .
$$

Proof. We modify the definition of $\mathbf{g}_{h}$ given above in some node $B_{k}$. For simplicity, let us choose this node different from all the vertices and their neighbors, and such that $h_{k}$ is comparable to $h$. For each $j$, let $\Gamma_{B_{j}}$ be the union of the two segments of $\mathcal{T}_{\Gamma, h}$ containing $B_{j}$. Moreover, we set $\Gamma_{V}=\cup_{i=1}^{N_{e}} \Gamma_{A_{i}}$.

We want to define $\mathbf{g}_{h}\left(B_{k}\right)$ in such a way that

$$
0=\int_{\Gamma} \mathbf{g}_{h} \cdot \mathbf{n}=\int_{\Gamma \backslash\left(\Gamma_{V} \cup \Gamma_{B_{k}}\right)} \mathbf{g}_{h} \cdot \mathbf{n}+\int_{\Gamma_{V}} \mathbf{g}_{h} \cdot \mathbf{n}+\int_{\Gamma_{B_{k}}} \mathbf{g}_{h} \cdot \mathbf{n},
$$

or, equivalently,

$$
\int_{\Gamma_{B_{k}}} \mathbf{g}_{h} \cdot \mathbf{n}=-\int_{\Gamma \backslash\left(\Gamma_{V} \cup \Gamma_{B_{k}}\right)} \mathbf{g}_{h} \cdot \mathbf{n}-\int_{\Gamma_{V}} \mathbf{g}_{h} \cdot \mathbf{n}=-\int_{\Gamma \backslash \Gamma_{B_{k}}} \mathbf{g}_{h} \cdot \mathbf{n} .
$$

But

$$
\begin{aligned}
\int_{\Gamma_{B_{k}}} \mathbf{g}_{h} \cdot \mathbf{n} & =\frac{1}{2} h_{k-1}\left[\mathbf{g}\left(B_{k-1}\right)+\mathbf{g}_{h}\left(B_{k}\right)\right] \cdot \mathbf{n}+\frac{1}{2} h_{k}\left[\mathbf{g}_{h}\left(B_{k}\right)+\mathbf{g}\left(B_{k+1}\right)\right] \cdot \mathbf{n} \\
& =\frac{1}{2}\left[h_{k-1} \mathbf{g}\left(B_{k-1}\right)+h_{k} \mathbf{g}\left(B_{k+1}\right)\right] \cdot \mathbf{n}+\frac{1}{2}\left(h_{k-1}+h_{k}\right) \mathbf{g}_{h}\left(B_{k}\right) \cdot \mathbf{n} .
\end{aligned}
$$

We introduce

$$
L_{1}(\mathbf{g})=-\int_{\Gamma \backslash \Gamma_{B_{k}}} \mathbf{g}_{h} \cdot \mathbf{n}-\frac{1}{2}\left[h_{k-1} \mathbf{g}\left(B_{k-1}\right)+h_{k} \mathbf{g}\left(B_{k+1}\right)\right] \cdot \mathbf{n} .
$$


Notice that the integral $\int_{\Gamma \backslash \Gamma_{B}} \mathbf{g}_{h} \cdot \mathbf{n}$ appears in the definition of $L_{1}$. Actually, $\mathbf{g}_{h}$ has been already defined in all the boundary nodes except for $B_{k}$ using the values of $\mathbf{g}$. Hence the notation $L_{1}(\mathbf{g})$ is consistent.

We define the value $\mathbf{g}_{h}\left(B_{k}\right)$ such that

$$
\begin{aligned}
& \frac{1}{2}\left(h_{k-1}+h_{k}\right) \mathbf{g}_{h}\left(B_{k}\right) \cdot \mathbf{n}=L_{1}(\mathbf{g}) \\
& \frac{1}{2}\left(h_{k-1}+h_{k}\right) \mathbf{g}_{h}\left(B_{k}\right) \cdot \mathbf{t}=0
\end{aligned}
$$

where $\mathbf{t}$ denotes the unit tangential vector on $\Gamma$. Taking into account that $\mathbf{g}$ satisfies the compatibility condition, we have

$$
\begin{aligned}
L_{1}(\mathbf{g})= & \int_{\Gamma \backslash\left(\Gamma_{V} \cup \Gamma_{B_{k}}\right)}\left(\mathbf{g}-\mathbf{g}_{h}\right) \cdot \mathbf{n}+\int_{\Gamma_{V}}\left(\mathbf{g}-\mathbf{g}_{h}\right) \cdot \mathbf{n}+\int_{\Gamma_{B_{k}}} \mathbf{g} \cdot \mathbf{n} \\
& -\frac{1}{2}\left[h_{k-1} \mathbf{g}\left(B_{k-1}\right)+h_{k} \mathbf{g}\left(B_{k+1}\right)\right] \cdot \mathbf{n} .
\end{aligned}
$$

The first term can be bounded using standard results for interpolation errors on $\Gamma \backslash\left(\Gamma_{V} \cup \Gamma_{B_{k}}\right)$. To bound the other three terms, we use that $\|\mathbf{g}\|_{L^{\infty}(\Gamma)} \leq\|\mathbf{g}\|_{1, \Gamma}$ and that the length of the integration set is less than $h$. Then we obtain

$$
\left|L_{1}(\mathbf{g})\right| \leq C h\|\mathbf{g}\|_{1, \Gamma} .
$$

It is easy to check that the matrix of the system (3.4) (for $\mathbf{g}_{h}\left(B_{k}\right)$ ) is nonsingular and its inverse has norm of order $h^{-1}$. So that we have

$$
\left|\mathbf{g}_{h}\left(B_{k}\right)\right| \leq C\|\mathbf{g}\|_{1, \Gamma},
$$

where $\left|\mathbf{g}_{h}\left(B_{k}\right)\right|$ stands for the Euclidean norm of the vector $\mathbf{g}_{h}\left(B_{k}\right)$. Therefore $\mathbf{g}_{h}$ is defined on the entire $\Gamma$ and satisfies the compatibility condition and the bound (3.3).

In the proof of the next proposition, we will use the embedding inequality for $0 \leq s<\frac{1}{2}$

$$
\|\phi\|_{L^{q}(\Gamma)} \leq C_{s}\|\phi\|_{s, \Gamma}, \quad \forall \phi \in H^{s}(\Gamma)
$$

with $q=\frac{2}{1-2 s}$ and

$$
C_{s} \sim \sqrt{\frac{1}{1-2 s}} \quad \text { when } s \rightarrow\left(\frac{1}{2}\right)^{-} .
$$

Inequality (3.7) is proved in [11, Theorem 1.1] in $\mathbb{R}$. The analogous result follows for an interval, and therefore for $\Gamma$, by using an extension theorem.

Proposition 3.1. For all $0 \leq s<\frac{1}{2}$ we have

$$
\left\|\mathbf{g}-\mathbf{g}_{h}\right\|_{-s, \Gamma} \leq \frac{C}{\sqrt{1-2 s}} h^{\frac{1}{2}+s}\|\mathbf{g}\|_{1, \Gamma}
$$


Proof. Let us set $p=\frac{2}{1+2 s}$ and $q=\frac{2}{1-2 s}$ its dual exponent. Using the Hölder inequality and the embedding inequality (3.7), we have

$$
\begin{aligned}
\left\|\mathbf{g}-\mathbf{g}_{h}\right\|_{-s, \Gamma} & =\sup _{\phi:\|\phi\|_{s, \Gamma}=1} \int_{\Gamma}\left(\mathbf{g}-\mathbf{g}_{h}\right) \phi \\
& \leq \sup _{\phi: \mid \phi \|_{s, \Gamma}=1}\left\|\mathbf{g}-\mathbf{g}_{h}\right\|_{L^{p}(\Gamma)}\|\phi\|_{L^{q}(\Gamma)} \\
& \leq C_{s}\left\|\mathbf{g}-\mathbf{g}_{h}\right\|_{L^{p}(\Gamma)} .
\end{aligned}
$$

Since $\mathbf{g}_{h}$ coincides with the Lagrange interpolation of $\mathbf{g}$ on $\Gamma \backslash\left(\Gamma_{V} \cup \Gamma_{B_{k}}\right), \mid \Gamma_{V} \cup$ $\Gamma_{B_{k}} \mid \leq C h$, and $1<p<2$, we have

$$
\begin{aligned}
\left\|\mathbf{g}-\mathbf{g}_{h}\right\|_{L^{p}(\Gamma)}^{p} & =\left\|\mathbf{g}-\mathbf{g}_{h}\right\|_{L^{p}\left(\Gamma \backslash\left(\Gamma_{V} \cup \Gamma_{B_{k}}\right)\right)}^{p}+\left\|\mathbf{g}-\mathbf{g}_{h}\right\|_{L^{p}\left(\Gamma_{V} \cup \Gamma_{B_{k}}\right)}^{p} \\
& \leq C h^{p}\|\mathbf{g}\|_{W^{1, p}\left(\Gamma \backslash\left(\Gamma_{V} \cup \Gamma_{B_{k}}\right)\right)}^{p}+C h\|\mathbf{g}\|_{L^{\infty}\left(\Gamma_{V} \cup \Gamma_{B_{k}}\right)}^{p} \\
& \leq C h \sum_{i=1}^{N_{e}}\|\mathbf{g}\|_{H^{1}\left(\Gamma_{i}\right)}^{p}
\end{aligned}
$$

which together with (3.9) yields,

$$
\left\|\mathbf{g}-\mathbf{g}_{h}\right\|_{-s, \Gamma} \leq C C_{s} h^{\frac{1}{p}}\|\mathbf{g}\|_{1, \Gamma} .
$$

Using (3.8) and recalling that $p=\frac{2}{1+2 s}$, we conclude the proof.

In the next proposition we obtain a quasi-uniform in $h$ estimate of the $H^{\frac{1}{2}}$-norm of $\mathbf{g}_{h}$.

Proposition 3.2. If the family of meshes $\mathcal{T}_{\Gamma, h}$ is quasi-uniform we have

$$
\left\|\mathbf{g}_{h}\right\|_{\frac{1}{2}, \Gamma} \leq C|\log h|\|\mathbf{g}\|_{1, \Gamma} .
$$

Proof. Let $\tilde{\mathbf{g}}_{h}$ the Carstensen approximation. Then, for $0<s<1 / 2$, inverse estimates imply

$$
\left\|\mathbf{g}_{h}\right\|_{\frac{1}{2}, \Gamma} \leq\left\|\mathbf{g}_{h}-\tilde{\mathbf{g}}_{h}\right\|_{\frac{1}{2}, \Gamma}+\left\|\tilde{\mathbf{g}}_{h}\right\|_{\frac{1}{2}, \Gamma} \leq C\left(h^{-\frac{1}{2}}\left\|\mathbf{g}_{h}-\tilde{\mathbf{g}}_{h}\right\|_{0, \Gamma}+h^{s-\frac{1}{2}}\left\|\tilde{\mathbf{g}}_{h}\right\|_{s, \Gamma}\right)
$$

and so, by (2.27) and the fact that $\mathbf{g} \in \mathbf{H}^{s}(\Gamma)$,

$$
\begin{aligned}
\left\|\mathbf{g}_{h}\right\|_{\frac{1}{2}, \Gamma} & \leq C\left(h^{-\frac{1}{2}}\left\|\mathbf{g}_{h}-\tilde{\mathbf{g}}_{h}\right\|_{0, \Gamma}+h^{s-\frac{1}{2}}\|\mathbf{g}\|_{s, \Gamma}\right) \\
& \leq C h^{-\frac{1}{2}}\left(\left\|\mathbf{g}_{h}-\mathbf{g}\right\|_{0, \Gamma}+\left\|\mathbf{g}-\tilde{\mathbf{g}}_{h}\right\|_{0, \Gamma}+h^{s}\|\mathbf{g}\|_{s, \Gamma}\right) .
\end{aligned}
$$

Using Proposition 3.1 (2.26), and (3.2), we obtain

$$
\left\|\mathbf{g}_{h}\right\|_{\frac{1}{2}, \Gamma} \leq C\left(\|\mathbf{g}\|_{1, \Gamma}+h^{s-\frac{1}{2}}\|\mathbf{g}\|_{s, \Gamma}\right) \leq C \frac{h^{s-\frac{1}{2}}}{1-2 s}\|\mathbf{g}\|_{1, \Gamma} .
$$

Choosing $s$ such that $1-2 s=1 /|\log h|$ we conclude the proof.

Remark 3.1. The quasi-uniformity assumption in the previous proposition is not essential. We have given the proof under this hypothesis for the sake of simplicity. However, for a general family of meshes, an elementary but rather technical computation using the definition of the fractional norm leads to the estimate

$$
\left\|\mathbf{g}_{h}\right\|_{\frac{1}{2}, \Gamma} \leq C\left|\log \left(h_{\text {min }}\right)\right|\|\mathbf{g}\|_{1, \Gamma} .
$$

where $h_{\text {min }}$ denotes the minimum mesh-size of $\mathcal{T}_{\Gamma, h}$. 


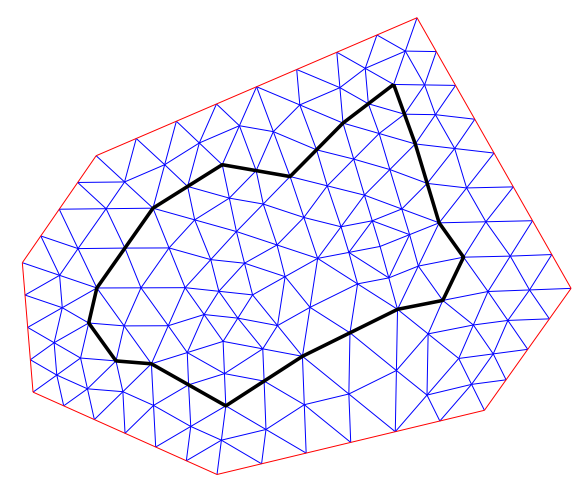

Figure 1. A face of $\Omega$, with its mesh and the polygonal $e_{0}$ in black.

3.2. Three dimensional case. We assume that the boundary $\Gamma$ is composed by $N_{e}$ polygonal faces $\Gamma_{i}$ and that $\left.\mathbf{g}\right|_{\Gamma_{i}} \in \mathbf{H}^{2}\left(\Gamma_{i}\right)$. Therefore $\mathbf{g} \in \mathbf{L}^{\infty}(\Gamma)$ and $\|\mathbf{g}\|_{L^{\infty}(\Gamma)} \leq C\|\mathbf{g}\|_{2, \Gamma}$. Moreover, we can show as in the two dimensional case, that $\mathbf{g} \in \mathbf{H}^{s}(\Gamma)$ for $0 \leq s<\frac{1}{2}$ and that

$$
\|\mathbf{g}\|_{s, \Gamma} \leq \frac{C}{1-2 s}\|\mathbf{g}\|_{2, \Gamma} .
$$

Assume that we have a triangular mesh $\mathcal{T}_{\Gamma, h}$ which is quasi-uniform. A construction, similar to the one proposed here, can be made also in the case of quadrilateral quasiuniform meshes.

As for the $2 \mathrm{D}$ case, let $\left\{B_{j}\right\}$ be the set of nodes of $\mathcal{T}_{\Gamma, h}$ and define

$$
E=\bigcup\{\bar{e}: e \text { is an edge of } \Omega\} .
$$

For each node $B_{j} \in E$, let us choose $T_{B_{j}}$ an element of $\mathcal{T}_{\Gamma, h}$ such that $B_{j} \in \bar{T}_{B_{j}}$. Finally let $e_{0}$ be a polygonal contained in a face $\Gamma_{k}$ of $\Omega$, with $\left|e_{0}\right|=O(1)$, made up of sides of triangles in $\mathcal{T}_{\Gamma, h}$ and such that triangles with a vertex on $e_{0}$ do not have vertices on $E$, see Fig. 1 for an example. It is clear that we can take it. We denote by $\mathbf{n}_{e_{0}}$ the normal vector to the face $\Gamma_{k}$ containing $e_{0}$.

Lemma 3.2. Given $\mathbf{g} \in \mathbf{L}^{2}(\Gamma)$ such that $\left.\mathbf{g}\right|_{\Gamma_{i}} \in \mathbf{H}^{2}\left(\Gamma_{i}\right)$ where $\Gamma_{i}$ for $i=1, \ldots, N_{e}$ are the faces of $\Gamma$, there exists a piecewise linear function $\mathbf{g}_{h} \in \mathbf{G}_{h}$ which is a modified Lagrange interpolant of $\mathbf{g}$ satisfying the compatibility condition (2.1) and

$$
\left\|\mathbf{g}_{h}\right\|_{L^{\infty}(\Gamma)} \leq C\|\mathbf{g}\|_{2, \Gamma} .
$$

Proof. We define the Lagrange interpolation $\mathbf{g}_{h} \in \mathbf{G}_{h}$ of $\mathbf{g}$ as the continuous piecewise linear function on $\mathcal{T}_{\Gamma, h}$ such that for each node $B_{j}$ in $\mathcal{T}_{\Gamma, h}$ we have

$$
\mathbf{g}_{h}\left(B_{j}\right)=\left\{\begin{array}{cl}
\mathbf{g}\left(B_{j}\right) & \text { if } B_{j} \notin\left(E \cup e_{0}\right) \\
\left.\mathbf{g}\right|_{T_{B_{j}}}\left(B_{j}\right) & \text { if } B_{j} \in E \\
\boldsymbol{\alpha} & \text { if } B_{j} \in e_{0},
\end{array}\right.
$$

where $\boldsymbol{\alpha}$ is a vector to be chosen in order to verify the compatibility condition (2.1). 
For a set $A \subset \Gamma$, we denote by $\omega_{\Gamma, A}$ the union of the closures of the elements in $\mathcal{T}_{\Gamma, h}$ having a vertex on the closure of $A$. Then we impose

$$
0=\int_{\Gamma} \mathbf{g}_{h} \cdot \mathbf{n}=\int_{\Gamma \backslash\left(\omega_{\Gamma, E} \cup \omega_{\Gamma, e_{0}}\right)} \mathbf{g}_{h} \cdot \mathbf{n}+\int_{\omega_{\Gamma, E}} \mathbf{g}_{h} \cdot \mathbf{n}+\int_{\omega_{\Gamma, e_{0}}} \mathbf{g}_{h} \cdot \mathbf{n} .
$$

Let us compute the last term. Clearly, $\omega_{\Gamma, e_{0}}$ lays on the face $\Gamma_{k}$ with normal $\mathbf{n}_{e_{0}}$. Each triangle $T$ in $\omega_{\Gamma, e_{0}}$ has $r_{T} \geq 1$ vertices on $\bar{e}_{0}$, that we denote $P_{T, 1}, \ldots, P_{T, r_{t}}$, while $P_{T, r_{T}+1}, \ldots, P_{T, 3}$ are the remaining ones. Then

$$
\int_{\omega_{\Gamma, e_{0}}} \mathbf{g}_{h} \cdot \mathbf{n}=\frac{1}{3} \sum_{T \subset \omega_{\Gamma, e_{0}}}|T| r_{T} \boldsymbol{\alpha} \cdot \mathbf{n}_{e_{0}}+\frac{1}{3} \sum_{T \subset \omega_{\Gamma, e_{0}}}|T| \sum_{i=r_{T}+1}^{3} \mathbf{g}_{h} \cdot \mathbf{n}_{e_{0}}\left(P_{T, i}\right)
$$

We require that the vector $\alpha$ is such that the following equality holds true

$$
\left(\frac{1}{3} \sum_{T \subset \omega_{\Gamma, e_{0}}}|T| r_{T}\right) \boldsymbol{\alpha} \cdot \mathbf{n}_{e_{0}}=L_{1}(\mathbf{g})
$$

where, taking into account that the continuous solution satisfies (1.3),

$$
\begin{aligned}
L_{1}(\mathbf{g}):=\int_{\Gamma \backslash\left(\omega_{\Gamma, E} \cup \omega_{\Gamma, e_{0}}\right)}\left(\mathbf{g}-\mathbf{g}_{h}\right) \cdot \mathbf{n}+\int_{\omega_{\Gamma, E}}\left(\mathbf{g}-\mathbf{g}_{h}\right) \cdot \mathbf{n} \\
\quad+\int_{\omega_{\Gamma, e_{0}}} \mathbf{g} \cdot \mathbf{n}-\frac{1}{3} \sum_{T \subset \omega_{\Gamma, e_{0}}}|T| \sum_{i=r_{T}+1}^{3} \mathbf{g}_{h} \cdot \mathbf{n}_{e_{0}}\left(P_{T, i}\right) .
\end{aligned}
$$

Since $\left|\omega_{\Gamma, E}\right|$ and $\left|\omega_{\Gamma, e_{0}}\right|$ are bounded by $C h$, using interpolation error estimates, we see that

$$
\left|L_{1}(\mathbf{g})\right| \leq C h\|\mathbf{g}\|_{2, \Gamma} .
$$

In order to be able to find a unique $\boldsymbol{\alpha}$, we add two conditions on the tangential components obtaining the following system

$$
\begin{aligned}
\left(\frac{1}{3} \sum_{T \subset \omega_{\Gamma, e_{0}}}|T| r_{T}\right) \boldsymbol{\alpha} \cdot \mathbf{n}_{e_{0}} & =L_{1}(\mathbf{g}) \\
\boldsymbol{\alpha} \cdot \mathbf{t}_{1} & =0 \\
\boldsymbol{\alpha} \cdot \mathbf{t}_{2} & =0
\end{aligned}
$$

where $\mathbf{t}_{1}$ and $\mathbf{t}_{\mathbf{2}}$ are unitary vectors such that together with $\mathbf{n}_{e_{0}}$ form an orthogonal basis of $\mathbb{R}^{3}$. This is a linear system for $\boldsymbol{\alpha}$ whose non singular matrix $M$ verifies $\left\|M^{-1}\right\| \leq C \frac{1}{h}$ since the mesh is quasi-uniform. Therefore, we can find $\boldsymbol{\alpha}$ such that

$$
|\boldsymbol{\alpha}| \leq C\|\mathbf{g}\|_{2, \Gamma} .
$$

This inequality, together with the definition of $\mathbf{g}_{h}$, gives (3.11).

In the following proposition we estimate $\left\|\mathbf{g}-\mathbf{g}_{h}\right\|_{-s, \Gamma}$. Since the best possible exponent $q$ in the embedding inequality (3.7) depends on the dimension, the argument used in Proposition 3.1 does not give an optimal result in the case of a three dimensional domain. We can give a different argument using a Hardy type inequality. It will become clear that the same argument can be used for $d=2$, but it gives a worse constant in terms of $s$ than that obtained in the Proposition 3.1 . 
Proposition 3.3. There exists a positive constant, such that, for all $0 \leq s<\frac{1}{2}$, the following bound holds true

$$
\left\|\mathbf{g}-\mathbf{g}_{h}\right\|_{-s, \Gamma} \leq \frac{C}{1-2 s} h^{\frac{1}{2}+s}\|\mathbf{g}\|_{2, \Gamma} .
$$

Proof. For each $\Gamma_{i}$, face of $\Omega$, and $x \in \Gamma_{i}$, we denote by $d_{i}(x)$ the distance of $x$ from $\partial \Gamma_{i}$. There exists a constant $C$ such that, for $0 \leq s<\frac{1}{2}$ and every $\phi \in H^{s}\left(\Gamma_{i}\right)$, we have

$$
\left\|\frac{\phi}{d_{i}^{s}}\right\|_{0, \Gamma_{i}} \leq \frac{C}{1-2 s}\|\phi\|_{s, \Gamma_{i}} .
$$

This estimate with a precise constant is proved in [7] for the half-space, by standard argument, one can show that the behavior of the constant in terms of $s$ is the same for Lipschitz bounded domains.

For simplicity let us assume that the polygonal $e_{0}$ chosen in the construction of $\mathbf{g}_{h}$ is close to the boundary of $\Gamma_{k}$, i.e., if $x \in e_{0}$, then $d_{k}(x) \leq C_{1} h$ for some constant $C_{1}$. Then, for any $\phi \in H^{s}(\Gamma)$,

$$
\int_{\Gamma}\left(\mathbf{g}-\mathbf{g}_{h}\right) \phi=\sum_{i=1}^{N_{e}} \int_{\Gamma_{i}}\left(\mathbf{g}-\mathbf{g}_{h}\right) \phi \leq \sum_{i=1}^{N_{e}}\left\|\left(\mathbf{g}-\mathbf{g}_{h}\right) d_{i}^{s}\right\|_{0, \Gamma_{i}}\left\|\frac{\phi}{d_{i}^{s}}\right\|_{0, \Gamma_{i}},
$$

and therefore, using (3.14), we obtain

$$
\left\|\mathbf{g}-\mathbf{g}_{h}\right\|_{-s, \Gamma}=\sup _{\phi:\|\phi\|_{s}=1} \int_{\Gamma}\left(\mathbf{g}-\mathbf{g}_{h}\right) \phi \leq \frac{C}{1-2 s} \sum_{i=1}^{N_{e}}\left\|\left(\mathbf{g}-\mathbf{g}_{h}\right) d_{i}^{s}\right\|_{0, \Gamma_{i}} .
$$

But,

$$
\begin{aligned}
\left\|\left(\mathbf{g}-\mathbf{g}_{h}\right) d_{i}^{s}\right\|_{0, \Gamma_{i}}^{2} & =\int_{\left\{x \in \Gamma_{i}: d_{i}(x) \leq C_{1} h\right\}}\left(\mathbf{g}-\mathbf{g}_{h}\right)^{2} d_{i}^{2 s}+\int_{\left\{x \in \Gamma_{i}: d_{i}(x)>C_{1} h\right\}}\left(\mathbf{g}-\mathbf{g}_{h}\right)^{2} d_{i}^{2 s} \\
& \leq C\left(h^{2 s+1}\|\mathbf{g}\|_{L^{\infty}(\Gamma)}^{2}+h^{2}\|\mathbf{g}\|_{1, \Gamma_{i}}^{2}\right) \quad \text { for } i \neq k, \\
\left\|\left(\mathbf{g}-\mathbf{g}_{h}\right) d_{k}^{s}\right\|_{0, \Gamma_{k}}^{2} & \leq C\left(h^{2 s+1}\|\mathbf{g}\|_{L^{\infty}(\Gamma)}^{2}+h^{2 s+1}\|\mathbf{g}\|_{2, \Gamma}^{2}+h^{2}\|\mathbf{g}\|_{1, \Gamma_{k}}^{2}\right)
\end{aligned}
$$

where, for the first term, we have used that $\left|\left\{x \in \Gamma_{i}: d_{i}(x) \leq C_{1} h\right\}\right| \leq C h$, that $\left\|\mathbf{g}_{h}\right\|_{L^{\infty}(\Gamma)} \leq C\|\mathbf{g}\|_{L^{\infty}(\Gamma)}$, and inequality (3.12), while, for the second one, that $\mathbf{g}_{h}$ agrees with the Lagrange interpolation. Hence, we conclude that, for all $0 \leq s<\frac{1}{2}$, the bound (3.13) holds true.

The next proposition can be proved using the same argument as in Proposition 3.2

Proposition 3.4. If the family of meshes $\mathcal{T}_{\Gamma, h}$ is quasi-uniform we have

$$
\left\|\mathbf{g}_{h}\right\|_{\frac{1}{2}, \Gamma} \leq C|\log h|\|\mathbf{g}\|_{2, \Gamma} .
$$

We are ready to prove the main theorem of the section.

Theorem 3.1. Let $\Omega \subset \mathbb{R}^{d}, d=2$ or 3 , be a convex polygonal or polyhedral domain. Suppose that $\left.\mathbf{g}\right|_{\Gamma_{i}} \in H^{d-1}\left(\Gamma_{i}\right)$ for all $\Gamma_{i}$ and that the family of meshes $\mathcal{T}_{\Gamma, h}$ is quasi-uniform. Let $\mathbf{g}_{h}$ be given by the modified Lagrange interpolation of $\mathbf{g}$ introduced in Lemmas 3.1 and 3.2. Then, we have 
(1) For $\Omega \subset \mathbb{R}^{2}$ a convex polygonal domain

$$
\left\|\mathbf{u}-\mathbf{u}_{h}\right\|_{0, \Omega}+\left\|p-p_{h}\right\|_{H^{-1}(\Omega) / \mathbb{R}} \leq C h|\log h|^{\frac{3}{2}}\|\mathbf{g}\|_{1, \Gamma},
$$

(2) For $\Omega \subset \mathbb{R}^{3}$ a convex polyhedral domain

$$
\left\|\mathbf{u}-\mathbf{u}_{h}\right\|_{0, \Omega}+\left\|p-p_{h}\right\|_{H^{-1}(\Omega) / \mathbb{R}} \leq C h|\log h|^{2}\|\mathbf{g}\|_{2, \Gamma} .
$$

Proof. From Propositions 2.23 .1 and 3.3 we have, for $0 \leq s<\frac{1}{2}$,

$$
\|\mathbf{u}-\mathbf{u}(h)\|_{0, \Omega}+\|p-p(h)\|_{H^{-1}(\Omega) / \mathbb{R}} \leq \frac{C}{(1-2 s)^{\frac{d+1}{2}}} h^{\frac{1}{2}+s}\|\mathbf{g}\|_{d-1, \Gamma} .
$$

Then, taking $s=1 / 2+1 / \log h<1 / 2$ yields

$$
\|\mathbf{u}-\mathbf{u}(h)\|_{0, \Omega}+\|p-p(h)\|_{H^{-1}(\Omega) / \mathbb{R}} \leq C h|\log h|^{\frac{d+1}{2}}\|\mathbf{g}\|_{d-1, \Gamma} .
$$

On the other hand, from Propositions 2.3, 3.2 and 3.4, we have

$$
\left\|\mathbf{u}(h)-\mathbf{u}_{h}\right\|_{0, \Omega}+\left\|p(h)-p_{h}\right\|_{H^{-1}(\Omega) / \mathbb{R}} \leq C h|\log h|\|\mathbf{g}\|_{d-1, \Gamma}
$$

which together with 3.15 gives the desired estimates.

Remark 3.2. In view of Remark [3.1, the quasi-uniformity assumption in the previous theorem can be removed obtaining, for general regular family of meshes, the analogous estimates with $|\log h|$ replaced by $\left|\log \left(h_{\text {min }}\right)\right|$.

\section{A POSTERIORI ERROR ESTIMATES}

In this section we introduce the error indicator for the finite element solution of our problem and show that it provides upper and lower bounds for the discretization error of the regularized problem.

We denote by $\mathcal{E}_{h}$ the union of the interior edges/faces of the elements of the mesh $\mathcal{T}_{h}$, and define

$$
\mathbf{J}: \mathcal{E}_{h} \rightarrow \mathbb{R}^{d},\left.\quad \mathbf{J}\right|_{e}=\mathbf{J}_{e} \quad \text { with } \mathbf{J}_{e}=\llbracket \frac{\partial \mathbf{u}_{h}}{\partial \mathbf{n}}-p_{h} \mathbf{n} \|_{e} \text { for } e \in \mathcal{E}_{h}
$$

where the jump of the function $r$ across the edge $e=T^{+} \cap T^{-}$is given by

$$
\llbracket \frac{\partial \mathbf{u}_{h}}{\partial \mathbf{n}}-p_{h} \mathbf{n} \rrbracket_{e}=\left(\frac{\left.\partial \mathbf{u}_{h}\right|_{T^{+}}}{\partial \mathbf{n}^{+}}-\left.p_{h}\right|_{T^{+}} \mathbf{n}^{+}\right)+\left(\frac{\left.\partial \mathbf{u}_{h}\right|_{T^{-}}}{\partial \mathbf{n}^{-}}-\left.p_{h}\right|_{T^{-}} \mathbf{n}^{-}\right)
$$

if $\mathbf{n}^{ \pm}$denotes the exterior normal to the triangle $T^{ \pm}$.

Then we introduce the local error indicator

$$
\eta_{T}^{2}=h_{T}^{4}\left\|-\Delta \mathbf{u}_{h}+\nabla p_{h}\right\|_{0, T}^{2}+h_{T}^{2}\left\|\operatorname{div} \mathbf{u}_{h}\right\|_{0, T}^{2}+\frac{1}{2} \sum_{e \subset \bar{T}} h_{T}^{3}\left\|\mathbf{J}_{e}\right\|_{0, e}^{2} .
$$

Since we want to estimate the velocity in the $L^{2}(\Omega)$-norm and the pressure in the $H^{-1}(\Omega) / \mathbb{R}$-norm, the error indicator results to be the usual error indicator for problems with smooth boundary data multiplied by $h_{T}^{2}$ (see, e.g., [23, 15]).

Proposition 4.1 (Robustness). The estimator $\eta_{T}$ introduced in (4.1) is robust, that is, there exists a positive constant $C$ independent of $h$ such that

$$
\left\|\mathbf{u}(h)-\mathbf{u}_{h}\right\|_{0, \Omega}+\left\|p(h)-p_{h}\right\|_{H^{-1}(\Omega) / \mathbb{R}} \leq C\left(\sum_{T \in \mathcal{T}_{h}} \eta_{T}^{2}\right)^{\frac{1}{2}} .
$$


Proof. We start with the estimate for $\mathbf{u}(h)-\mathbf{u}_{h}$. In order to apply a duality argument, we consider the solution $(\phi, q)$ of (2.19). Then, taking into account the equations (2.18) and (2.5), and the approximation estimates (2.14) and (2.15), we obtain by integration by parts:

(4.3)

$$
\begin{aligned}
\left\|\mathbf{u}(h)-\mathbf{u}_{h}\right\|_{0, \Omega}^{2}= & \left(\mathbf{u}(h)-\mathbf{u}_{h}, \mathbf{u}(h)-\mathbf{u}_{h}\right)=\left(\mathbf{u}(h)-\mathbf{u}_{h},-\Delta \phi+\nabla q\right) \\
= & \left(\nabla\left(\mathbf{u}(h)-\mathbf{u}_{h}\right), \nabla\left(\phi-\phi^{I}\right)\right) \\
& \quad-\left(\operatorname{div}\left(\mathbf{u}(h)-\mathbf{u}_{h}\right), q-P_{0} q\right)-\left(p(h)-p_{h}, \operatorname{div}\left(\phi-\phi^{I}\right)\right. \\
= & \sum_{T \in \mathcal{T}_{h}}\left(\left(\Delta \mathbf{u}_{h}, \boldsymbol{\phi}-\boldsymbol{\phi}^{I}\right)_{T}-\left(\frac{\partial \mathbf{u}_{h}}{\partial \mathbf{n}}, \boldsymbol{\phi}-\boldsymbol{\phi}^{I}\right)_{\partial T}\right) \\
& \quad+\left(\operatorname{div} \mathbf{u}_{h}, q-P_{0} q\right) \\
& \quad-\sum_{T \in \mathcal{T}_{h}}\left(\left(\nabla p_{h}, \boldsymbol{\phi}-\boldsymbol{\phi}^{I}\right)_{T}-\left(p_{h},\left(\boldsymbol{\phi}-\boldsymbol{\phi}^{I}\right) \cdot \mathbf{n}\right)_{\partial T}\right) \\
= & -\sum_{T \in \mathcal{T}_{h}}\left(-\Delta \mathbf{u}_{h}+\nabla p_{h}, \boldsymbol{\phi}-\boldsymbol{\phi}^{I}\right)_{T}+\sum_{T \in \mathcal{T}_{h}}\left(\operatorname{div} \mathbf{u}_{h}, q-P_{0} q\right)_{T} \\
& -\sum_{e \in \mathcal{E}_{h}}\left(\llbracket \frac{\partial \mathbf{u}_{h}}{\partial \mathbf{n}}-p_{h} \mathbf{n} \rrbracket, \boldsymbol{\phi}-\boldsymbol{\phi}^{I}\right)_{e}
\end{aligned}
$$

Thanks to (2.14), we can write

$$
\begin{aligned}
\left\|\mathbf{u}(h)-\mathbf{u}_{h}\right\|_{0, \Omega}^{2} \leq & C \sum_{T \in \mathcal{T}_{h}}\left\|-\Delta \mathbf{u}_{h}+\nabla p_{h}\right\|_{0, T} h_{T}^{2}|\boldsymbol{\phi}|_{2, T} \\
& +C \sum_{T \in \mathcal{T}_{h}}\left\|\operatorname{div} \mathbf{u}_{h}\right\|_{0, T} h_{T}|q|_{1, T}+C \sum_{e \in \mathcal{E}_{h}}\|\mathbf{J}\|_{0, e} h_{T}^{\frac{3}{2}}|\boldsymbol{\phi}|_{2, \omega_{e}} \\
\leq & C\left[\sum_{T \in \mathcal{T}_{h}}\left(h_{T}^{4}\left\|-\Delta \mathbf{u}_{h}+\nabla p_{h}\right\|_{0, T}^{2}+h_{T}^{2}\left\|\operatorname{div} \mathbf{u}_{h}\right\|_{0, T}^{2}\right)\right. \\
& \left.+\sum_{e \in \mathcal{E}_{h}} h_{T}^{3}\|\mathbf{J}\|_{0, e}^{2}\right]^{\frac{1}{2}}\left\|\mathbf{u}(h)-\mathbf{u}_{h}\right\|_{0, \Omega} \\
\leq & C\left(\sum_{T \in \mathcal{T}_{h}} \eta_{T}^{2}\right)^{\frac{1}{2}}\left\|\mathbf{u}(h)-\mathbf{u}_{h}\right\|_{0, \Omega}
\end{aligned}
$$

where $\omega_{e}$ is the union ot the elements sharing $e \in \mathcal{E}_{h}$. This concludes the estimate of $\left\|\mathbf{u}(h)-\mathbf{u}_{h}\right\|_{0, \Omega}$.

Now we consider the error for the pressure. Since $p(h)$ and $p_{h}$ have zero mean value, the definition of the $H^{-1}$-norm reads

$$
\left\|p(h)-p_{h}\right\|_{H^{-1}(\Omega) / \mathbb{R}}=\sup _{\substack{q \in H_{0}^{1}(\Omega) \\ \int_{\Omega} q=0}} \frac{\left(p(h)-p_{h}, q\right)}{\|q\|_{1, \Omega}} .
$$


For each $q \in H_{0}^{1}(\Omega)$ with $\int_{\Omega} q=0$, we take $\psi \in H_{0}^{2}(\Omega)$ with $\operatorname{div} \psi=q$ and $\|\boldsymbol{\psi}\|_{H^{2}(\Omega)} \leq C\|q\|_{H^{1}(\Omega)}$ see (2.22), hence

$$
\begin{aligned}
\left(p(h)-p_{h}, q\right)= & \left(p(h)-p_{h}, \operatorname{div} \boldsymbol{\psi}\right) \\
= & \left(p(h)-p_{h}, \operatorname{div}\left(\boldsymbol{\psi}-\boldsymbol{\psi}^{I}\right)\right)-\left(\nabla\left(\mathbf{u}(h)-\mathbf{u}_{h}\right), \nabla\left(\boldsymbol{\psi}-\boldsymbol{\psi}^{I}\right)\right) \\
& \quad-\left(\mathbf{u}(h)-\mathbf{u}_{h}, \Delta \boldsymbol{\psi}\right) .
\end{aligned}
$$

By the same computations performed in equation (4.3), we obtain

$$
\begin{aligned}
\left(p(h)-p_{h}, q\right)=( & \left.\Delta \mathbf{u}(h)-\nabla p(h), \boldsymbol{\psi}-\boldsymbol{\psi}^{I}\right) \\
& +\sum_{T \in \mathcal{T}_{h}}\left(-\left(\Delta \mathbf{u}_{h}, \boldsymbol{\psi}-\boldsymbol{\psi}^{I}\right)_{T}+\left(\frac{\partial \mathbf{u}_{h}}{\partial \mathbf{n}}, \boldsymbol{\psi}-\boldsymbol{\psi}^{I}\right)_{T}\right) \\
& +\sum_{T \in \mathcal{T}_{h}}\left(\left(\nabla p_{h}, \boldsymbol{\psi}-\boldsymbol{\psi}^{I}\right)_{T}-\left(p_{h},\left(\boldsymbol{\psi}-\boldsymbol{\psi}^{I}\right) \cdot \mathbf{n}\right)_{\partial T}\right) \\
& -\left(\mathbf{u}(h)-\mathbf{u}_{h}, \Delta \boldsymbol{\psi}\right) \\
= & \sum_{T \in \mathcal{T}_{h}}\left(-\Delta \mathbf{u}_{h}+\nabla p_{h}, \boldsymbol{\psi}-\boldsymbol{\psi}^{I}\right)_{T} \\
& +\sum_{e \in \mathcal{E}_{h}}\left(\llbracket \frac{\partial \mathbf{u}_{h}}{\partial \mathbf{n}}-p_{h} \mathbf{n} \rrbracket, \boldsymbol{\psi}-\boldsymbol{\psi}^{I}\right)_{e}-\left(\mathbf{u}(h)-\mathbf{u}_{h}, \Delta \boldsymbol{\psi}\right) .
\end{aligned}
$$

Then

$$
\begin{aligned}
\left(p(h)-p_{h}, q\right) \leq C & {\left[\sum_{T \in \mathcal{T}_{h}} h_{T}^{4}\left\|-\Delta \mathbf{u}_{h}+\nabla p_{h}\right\|_{0, T}^{2}+\sum_{e \in \mathcal{E}_{h}} h_{T}^{3}\|\mathbf{J}\|_{0, e}^{2}\right]^{\frac{1}{2}}\|q\|_{1, \Omega} } \\
& +\left\|\mathbf{u}(h)-\mathbf{u}_{h}\right\|_{0, \Omega}\|q\|_{1, \Omega} .
\end{aligned}
$$

The proof concludes by using the estimate (4.4) and the norm definition (4.5).

In the next proposition we show that the error indicator bounds locally the error by below.

Proposition 4.2 (Efficiency). For all element $T \in \mathcal{T}_{h}$, we have

$$
\eta_{T} \leq C\left(\left\|\mathbf{u}(h)-\mathbf{u}_{h}\right\|_{0, \omega_{T}}+\left\|p(h)-p_{h}\right\|_{-1, \omega_{T}}\right)
$$

where $\omega_{T}=\left\{T^{\prime} \in \mathcal{T}_{h}: \overline{T^{\prime}} \cap \bar{T} \neq \emptyset\right\}$.

Proof. We estimate the three terms of the error indicator in (4.1), separately. Given an element $T \in \mathcal{T}_{h}$, let us consider the function

$$
b_{T}=\left(\prod_{i=1}^{d+1} \lambda_{i, T}\right)^{2}
$$

with $\lambda_{i, T}, i=1, \ldots, d+1$ being the barycenter coordinate functions in $T$. We set

$$
\mathbf{w}_{T}=\left(-\Delta \mathbf{u}_{h}+\nabla p_{h}\right) b_{T} .
$$

Thanks to the definition of $b_{T}$ we have that

$$
\mathbf{w}_{T}=0 \quad \text { on } \partial T, \quad \nabla \mathbf{w}_{T}=0 \quad \text { on } \partial T,
$$


and, by inverse inequality,

$$
\begin{aligned}
\left\|\operatorname{div} \mathbf{w}_{T}\right\|_{1, T} & \leq C h_{T}^{-2}\left\|-\Delta \mathbf{u}_{h}+\nabla p_{h}\right\|_{0, T} \\
\left\|\Delta \mathbf{w}_{T}\right\|_{0, T} & \leq C h_{T}^{-2}\left\|-\Delta \mathbf{u}_{h}+\nabla p_{h}\right\|_{0, T},
\end{aligned}
$$

Then integration by parts gives

$$
\begin{aligned}
\left\|-\Delta \mathbf{u}_{h}+\nabla p_{h}\right\|_{0, T}^{2} & \left.=\left(-\Delta \mathbf{u}_{h}+\nabla p_{h},-\Delta \mathbf{u}_{h}+\nabla p_{h}\right)\right)_{T} \\
& \leq C\left|\left(-\Delta \mathbf{u}_{h}+\nabla p_{h}, \mathbf{w}_{T}\right)_{T}\right| \\
& =C\left|\left(-\Delta\left(\mathbf{u}_{h}-\mathbf{u}(h)\right)+\nabla\left(p_{h}-p(h)\right), \mathbf{w}_{T}\right)\right| \\
& =C\left|\left(\mathbf{u}_{h}-\mathbf{u}(h), \Delta \mathbf{w}_{T}\right)_{T}+\left(p_{h}-p(h), \operatorname{div} \mathbf{w}_{T}\right)\right| .
\end{aligned}
$$

Due to the definition of $b_{T}$ we have that $\operatorname{div} \mathbf{w}_{T} \in \mathbf{H}_{0}^{1}(T)$, hence we can use the duality between $H^{-1}(T)$ and $H_{0}^{1}(T)$ to obtain

$\left\|-\Delta \mathbf{u}_{h}+\nabla p_{h}\right\|_{0, T}^{2} \leq C\left(\left\|\mathbf{u}_{h}-\mathbf{u}(h)\right\|_{0, T}\left\|\Delta \mathbf{w}_{T}\right\|_{0, T}+\left\|\left(p_{h}-p(h)\right)\right\|_{-1, T}\left\|\operatorname{div} \mathbf{w}_{T}\right\|_{1, T}\right)$

which, together with (4.7), implies

$$
h_{T}^{2}\left\|-\Delta \mathbf{u}_{h}+\nabla p_{h}\right\|_{0, T} \leq C\left(\left\|\mathbf{u}_{h}-\mathbf{u}(h)\right\|_{0, T}+\left\|p_{h}-p(h)\right\|_{-1, T}\right) .
$$

In order to bound the second term in (4.1), let us introduce $w_{T}=\left(\operatorname{div} \mathbf{u}_{h}\right) b_{T}$, which satisfies

$$
\left\|\nabla w_{T}\right\|_{0, T} \leq C h_{T}^{-1}\left\|\operatorname{div} \mathbf{u}_{h}\right\|_{0, T} .
$$

Hence we obtain

$$
\begin{aligned}
\int_{T}\left(\operatorname{div} \mathbf{u}_{h}\right)^{2} & \leq C\left|\int_{T}\left(\operatorname{div} \mathbf{u}_{h}\right) w_{T}\right|=C\left|\int_{T} \operatorname{div}\left(\mathbf{u}_{h}-\mathbf{u}(h)\right) w_{T}\right| \\
& =C\left|\int_{T}\left(\mathbf{u}_{h}-\mathbf{u}(h)\right) \nabla w_{T}\right| \leq C h_{T}^{-1}\left\|\mathbf{u}_{h}-\mathbf{u}(h)\right\|_{0, T}\left\|\operatorname{div} \mathbf{u}_{h}\right\|_{0, T}
\end{aligned}
$$

which implies

$$
h_{T}\left\|\operatorname{div} \mathbf{u}_{h}\right\|_{0, T} \leq C\left\|\mathbf{u}_{h}-\mathbf{u}(h)\right\|_{0, T}
$$

It remains to bound the last term of the indicator involving the jumps along element interfaces in $\mathcal{T}_{h}$. Let $e \in \mathcal{E}_{h}$ be an internal edge/face and let us suppose that there are two elements $T_{1}$ and $T_{2}$ such that $e=T_{1} \cap T_{2}$. Let $\mathrm{v}_{\mathrm{i}}$ for $i=1, \ldots, d$, be the vertices of $e$. We denote by $\lambda_{\mathrm{v}_{\mathrm{i}}, \mathrm{T}_{j}}, i=1, \ldots, d, j=1,2$, the barycentric coordinate functions for the vertex $\mathrm{v}_{i}$ on the triangle $T_{j}$ and by $\omega_{e}$ the union of $T_{1}$ and $T_{2}$. Then we define the bubble function

$$
b_{e}=\left(\prod_{i=1}^{d} \lambda_{\mathrm{v}_{i}, T_{1}} \prod_{i=1}^{d} \lambda_{\mathrm{v}_{i}, T_{2}}\right)^{2} .
$$

Setting $\mathbf{w}_{e}=\mathbf{J}_{e} b_{e}$ and taking into account that the mesh is regular, it is not difficult to check that the following inequalities hold true:

$$
\begin{aligned}
& \left\|\Delta \mathbf{w}_{e}\right\|_{0, \omega_{e}} \leq C h_{e}^{-\frac{3}{2}}\left\|\mathbf{J}_{e}\right\|_{0, e} \\
& \left\|\operatorname{div} \mathbf{w}_{e}\right\|_{1, \omega_{e}} \leq C h_{e}^{-\frac{3}{2}}\left\|\mathbf{J}_{e}\right\|_{0, e} \\
& \left\|\mathbf{w}_{e}\right\|_{0, \omega_{e}} \leq C h_{e}^{\frac{1}{2}}\left\|\mathbf{J}_{e}\right\|_{0, e} .
\end{aligned}
$$


There exists a positive constant $C$ such that

$$
\begin{aligned}
\frac{1}{C}\left\|\mathbf{J}_{e}\right\|_{0, e}^{2} \leq & \left(\mathbf{J}_{e}^{2}, b_{e}\right)_{e}=\left(\| \frac{\partial \mathbf{u}_{h}}{\partial \mathbf{n}}-p_{h} \mathbf{n} \rrbracket, \mathbf{w}_{e}\right)_{e} \\
= & \left(\nabla \mathbf{u}_{h}, \nabla \mathbf{w}_{e}\right)_{\omega_{e}}+\sum_{T \subset \omega_{e}}\left(\Delta \mathbf{u}_{h}, \mathbf{w}_{e}\right)_{T}-\sum_{T \subset \omega_{e}}\left(\nabla p_{h}, \mathbf{w}_{e}\right)_{T}-\left(p_{h}, \operatorname{div} \mathbf{w}_{e}\right)_{\omega_{e}} \\
= & \left(\nabla \mathbf{u}_{h}, \nabla \mathbf{w}_{e}\right)_{\omega_{e}}-\left(p_{h}, \operatorname{div} \mathbf{w}_{e}\right)_{\omega_{e}}+\sum_{T \subset \omega_{e}}\left(\Delta \mathbf{u}_{h}-\nabla p_{h}, \mathbf{w}_{e}\right)_{T} \\
= & \left(\nabla \mathbf{u}_{h}-\nabla \mathbf{u}(h), \nabla \mathbf{w}_{e}\right)_{\omega_{e}}-\left(p_{h}-p(h), \operatorname{div} \mathbf{w}_{e}\right)_{\omega_{e}} \\
& \quad+\sum_{T \subset \omega_{e}}\left(\Delta \mathbf{u}_{h}-\nabla p_{h}, \mathbf{w}_{e}\right)_{T} \\
= & -\left(\mathbf{u}_{h}-\mathbf{u}(h), \Delta \mathbf{w}_{e}\right)_{\omega_{e}}+\left(\mathbf{u}_{h}-\mathbf{u}(h), \frac{\partial \mathbf{w}_{e}}{\partial \mathbf{n}}\right)_{\partial \omega_{e}} \\
& \quad-\left(p_{h}-p(h), \operatorname{div} \mathbf{w}_{e}\right)_{\omega_{e}}+\sum_{T \subset \omega_{e}}\left(\Delta \mathbf{u}_{h}-\nabla p_{h}, \mathbf{w}_{e}\right)_{T} .
\end{aligned}
$$

Using again the fact that $\operatorname{div} \mathbf{w}_{e} \in H_{0}^{1}\left(\omega_{e}\right)$, we obtain, by multiplying times $h_{e}^{3}$

$$
\begin{aligned}
h_{e}^{3}\left\|\mathbf{J}_{e}\right\|_{0, e}^{2} \leq & C\left(\left\|\mathbf{u}_{h}-\mathbf{u}(h)\right\|_{0, \omega_{e}} h_{e}^{3}\left\|\Delta \mathbf{w}_{e}\right\|_{0, \omega_{e}}+\left\|p_{h}-p(h)\right\|_{-1, \omega_{e}} h_{e}^{3}\left\|\operatorname{div} \mathbf{w}_{e}\right\|_{1, \omega_{e}}\right. \\
& \left.+\sum_{T \subset \omega_{e}} h_{e}^{2}\left\|\Delta \mathbf{u}_{h}-\nabla p_{h}\right\|_{0, T} h_{e}\left\|\mathbf{w}_{e}\right\|_{0, T}\right) .
\end{aligned}
$$

Using (4.11) and (4.9), we have

$$
\begin{aligned}
h_{e}^{3}\left\|\mathbf{J}_{e}\right\|_{0, e}^{2} \leq & C\left(\left\|\mathbf{u}_{h}-\mathbf{u}(h)\right\|_{0, \omega_{e}}+\left\|p_{h}-p(h)\right\|_{-1, \omega_{e}}\right. \\
& \left.+\sum_{T \subset \omega_{e}} h_{e}^{2}\left\|\Delta \mathbf{u}_{h}-\nabla p_{h}\right\|_{0, T}\right) h_{e}^{\frac{3}{2}}\|\mathbf{J}\|_{0, e} \\
\leq & \left(\left\|\mathbf{u}_{h}-\mathbf{u}(h)\right\|_{0, \omega_{e}}+\left\|p_{h}-p(h)\right\|_{-1, \omega_{e}}\right) h_{e}^{\frac{3}{2}}\|\mathbf{J}\|_{0, e}
\end{aligned}
$$

that is,

$$
h_{e}^{\frac{3}{2}}\left\|\mathbf{J}_{e}\right\|_{0, e} \leq C\left(\left\|\mathbf{u}_{h}-\mathbf{u}(h)\right\|_{0, \omega_{e}}+\left\|p_{h}-p(h)\right\|_{-1, \omega_{e}}\right) .
$$

Taking into account the definition (4.1) of the estimator $\eta_{T}$, together with the estimates (4.9), (4.10) and (4.12) we obtain the desired result.

\section{Numerical Example}

On $\Omega=[0,1]^{2}$, we consider the lid-driven cavity flow problem

$$
\begin{aligned}
& -\Delta \mathbf{u}+\nabla p=0 \quad \text { in } \Omega \\
& \operatorname{div} \mathbf{u}=0 \quad \text { on } \Omega \\
& \mathbf{u}=\mathbf{g} \quad \text { in } \partial \Omega
\end{aligned}
$$

with

$$
\mathbf{g}\left(x_{1}, x_{2}\right)= \begin{cases}(1,0) & \text { if } 0<x_{1}<1 \text { and } x_{2}=1 \\ (0,0) & \text { if } x_{1}=0 \text { or } x_{1}=1 \text { or } x_{2}=0 .\end{cases}
$$

We consider the methods:

- Mini-element: $\mathbf{V}_{h}=\left(\mathcal{P}_{1}^{b}\left(\mathcal{T}_{h}\right)\right)^{2} \cap \mathcal{C}^{0}(\bar{\Omega})^{2}$ and $Q_{h}=\mathcal{P}_{1}\left(\mathcal{T}_{h}\right) \cap \mathcal{C}^{0}(\bar{\Omega}) \cap L_{0}^{2}(\Omega)$

- Hood and Taylor: $\mathbf{V}_{h}=\mathcal{P}_{2}\left(\mathcal{T}_{h}\right)^{2} \cap \mathcal{C}^{0}(\bar{\Omega})^{2}$ and $Q_{h}=\mathcal{P}_{1}\left(\mathcal{T}_{h}\right) \cap \mathcal{C}^{0}(\bar{\Omega}) \cap L_{0}^{2}(\Omega)$ 
where, if for an element $T, b_{T} \in \mathcal{P}_{3}$ is the cubic bubble function vanishing on $\partial T$, we set

$$
\mathcal{P}_{1}^{b}(T)=\mathcal{P}_{1}(T) \oplus \operatorname{span}\left\{b_{T}(\cdot)\right\} .
$$

We consider the variational formulation (2.15) with $\mathbf{u}_{h}=E \mathbf{g}_{h}+\mathbf{u}_{0 h}$ where $\mathbf{u}_{0 h} \in \mathbf{V}_{h}$ and $\mathbf{g}_{h}$ is the Lagrange interpolation of $\mathbf{g}$ on the restriction of $\mathcal{T}_{h}$ to $\partial \Omega$. We remark that the compatibility condition (2.1) is automatically verified.

Below for the distinct methods and different refinement strategies we estimate the convergence errors for $\mathbf{u}$ in $L^{2}(\Omega)$-norm. Since we do not know the exact solution, the $L^{2}(\Omega)$-error is computed as the difference between the solutions obtained at two consecutive refinements.

\begin{tabular}{|c|c|c|c|c|}
\hline nv & $L^{2}$ error in $\mathbf{u}$ & $\eta$ & order in $\mathbf{u}$ & order in $\eta$ \\
\hline 289 & 0.051393 & 1.8518 & & \\
\hline 1089 & 0.025876 & 0.93123 & 0.51724 & 0.51818 \\
\hline 4225 & 0.012952 & 0.46698 & 0.51049 & 0.5091 \\
\hline 16641 & 0.0064768 & 0.23386 & 0.50553 & 0.50449 \\
\hline 66049 & 0.0032384 & 0.11703 & 0.50281 & 0.5022 \\
\hline
\end{tabular}

TABLE 1. Mini-element on uniformly refined structured meshes.

\begin{tabular}{|c|c|c|c|c|}
\hline nv & $L^{2}$ error in $\mathbf{u}$ & $\eta$ & order in $\mathbf{u}$ & order in $\eta$ \\
\hline 289 & 0.04065 & 3.603 & & \\
\hline 1089 & 0.020324 & 1.8041 & 0.52253 & 0.52142 \\
\hline 4225 & 0.010162 & 0.90276 & 0.51127 & 0.51068 \\
\hline 16641 & 0.0050809 & 0.45158 & 0.50563 & 0.50532 \\
\hline
\end{tabular}

TABLE 2. Hood-Taylor on uniformly refined structured meshes.

Tables 1 and 2 show results obtained by uniform refinements starting with a coarse mesh for Mini-element and Hood-Taylor methods respectively. We observe that, in both cases, order $\frac{1}{2}$ with respect to the number of elements (order 1 in $h$ ) is obtained for the error decay in $L^{2}(\Omega)$ of $\mathbf{u}$. Accordingly, the error estimator $\eta$ defined by

$$
\eta^{2}=\sum_{T \in \mathcal{T}_{h}} \eta_{T}^{2}
$$

with $\eta_{T}$ given by (4.1), decreases with the same order.

In Tables 3 and 4 we show the results obtained by an adaptive procedure using the a posteriori error estimator (5.1). The refinement process is standard: given $0<\theta<1$, a fixed parameter, suppose that $\mathcal{T}_{k}$ is the mesh in the $k$-step. If we enumerate the triangular elements such that $\mathcal{T}_{k}=\left\{T_{i}: i=1, \ldots, N_{e l}\right\}$ with $\eta_{T_{i}} \geq \eta_{T_{i+1}}$, let $N_{r e f, k}$ be the minimum integer such that

$$
\sum_{i=1}^{N_{r e f, k}} \eta_{T_{i}}^{2} \geq \theta \eta^{2} .
$$

Then, the mesh for the $k+1$-step is constructed in such a way that the elements $T_{i}, i=1, \ldots, N_{r e f, k}$ are refined. We report the $L^{2}(\Omega)$-error in $\mathbf{u}$ which, as before, 


\begin{tabular}{|c|c|c|c|c|}
\hline nv & $L^{2}$ error in $\mathbf{u}$ & $\eta$ & order in $\mathbf{u}$ & order in $\eta$ \\
\hline 84 & 0.04161 & 2.4824 & & \\
\hline 99 & 0.036736 & 1.5566 & 0.75833 & 2.8409 \\
\hline 107 & 0.017576 & 1.0568 & 9.4869 & 4.9828 \\
\hline 148 & 0.015596 & 0.67029 & 0.36853 & 1.4036 \\
\hline 201 & 0.010888 & 0.4429 & 1.174 & 1.3537 \\
\hline 316 & 0.0064777 & 0.27551 & 1.1477 & 1.0493 \\
\hline 514 & 0.0041708 & 0.17191 & 0.90498 & 0.96945 \\
\hline 778 & 0.0025481 & 0.11492 & 1.1888 & 0.9716 \\
\hline 1197 & 0.0017212 & 0.07334 & 0.91061 & 1.0425 \\
\hline 1901 & 0.0011844 & 0.04755 & 0.80805 & 0.93682 \\
\hline 2859 & 0.00074065 & 0.030819 & 1.1504 & 1.0626 \\
\hline 4416 & 0.00050373 & 0.01985 & 0.88666 & 1.0118 \\
\hline 6834 & 0.00033144 & 0.01284 & 0.95861 & 0.99762 \\
\hline 10248 & 0.00021719 & 0.0084216 & 1.0431 & 1.041 \\
\hline 15443 & 0.00014117 & 0.0055306 & 1.0507 & 1.0254 \\
\hline
\end{tabular}

TABLE 3. Adaptive scheme for the Mini-element method using the local estimators $\eta_{T}$. Parameter: $\theta=0.5$.

\begin{tabular}{|c|c|c|c|c|}
\hline nv & $L^{2}$ error in $\mathbf{u}$ & $\eta$ & order in $\mathbf{u}$ & order in $\eta$ \\
\hline 84 & 0.035974 & 4.2984 & & \\
\hline 91 & 0.02702 & 2.3946 & 3.5759 & 7.3089 \\
\hline 102 & 0.015866 & 1.3891 & 4.6655 & 4.772 \\
\hline 118 & 0.0082372 & 0.82406 & 4.4988 & 3.5837 \\
\hline 160 & 0.0045485 & 0.46452 & 1.9503 & 1.8826 \\
\hline 237 & 0.0024787 & 0.25907 & 1.5452 & 1.4862 \\
\hline 385 & 0.0014542 & 0.1406 & 1.0991 & 1.2597 \\
\hline 636 & 0.00078342 & 0.075834 & 1.2322 & 1.2299 \\
\hline 992 & 0.00042274 & 0.041933 & 1.3878 & 1.3328 \\
\hline 1615 & 0.00026069 & 0.022199 & 0.99186 & 1.3051 \\
\hline 2583 & 0.00012981 & 0.011858 & 1.4848 & 1.3352 \\
\hline 4154 & $7.3921 e-005$ & 0.0062463 & 1.1851 & 1.3491 \\
\hline 6665 & $3.9114 e-005$ & 0.0032513 & 1.3463 & 1.381 \\
\hline 10447 & $1.9238 e-005$ & 0.0017148 & 1.5788 & 1.4235 \\
\hline 16629 & $1.0136 e-005$ & 0.00089668 & 1.3785 & 1.3948 \\
\hline 26283 & $5.559 e-006$ & 0.00046444 & 1.3122 & 1.4371 \\
\hline 40802 & $2.7269 e-006$ & 0.00024283 & 1.6195 & 1.4744 \\
\hline 64222 & $1.3931 e-006$ & 0.00012827 & 1.4806 & 1.4069 \\
\hline
\end{tabular}

TABLE 4. Adaptive scheme for the Hood-Taylor method using the local estimators $\eta_{T}$. Parameter: $\theta=0.75$.

is computed in each step as the $L^{2}(\Omega)$-norm of the difference between the discrete solution obtained in the current step and in the previous one of the iterative process.

We observe that for both Mini-element and Hood-Taylor methods, the adaptive process recovers the expected optimal order of convergence in $\mathbf{u}$. In Figure 2 we 

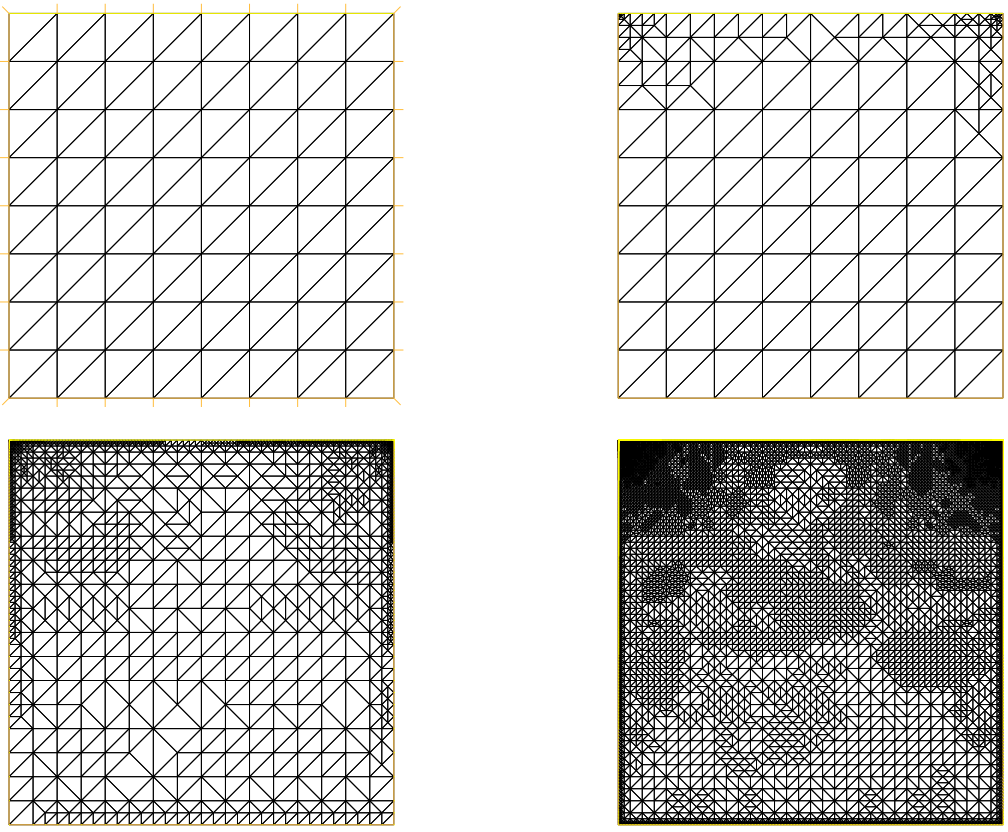

Figure 2. Sequence of meshes for the Hood-Taylor adaptive process using the local estimators $\eta_{T}$ with parameter $\theta=0.75$. Initial mesh and meshes of iterations 5, 10 and 15 .

show the initial mesh and some of the meshes obtained in the iterative process for Hood-Taylor method.

\section{ACKNOWLEDGMENTS}

We thank Pablo De Napoli who suggested us the argument used in Proposition 3.3, and Pedro Morin for helpful discussions.

\section{REFERENCES}

[1] R. A. Adams. Sobolev spaces. Academic Press [A subsidiary of Harcourt Brace Jovanovich, Publishers], New York-London, 1975. Pure and Applied Mathematics, Vol. 65.

[2] R.A. Adams and J.J.F. Fournier. Sobolev spaces, volume 140 of Pure and Applied Mathematics (Amsterdam). Elsevier/Academic Press, Amsterdam, second edition, 2003.

[3] H. Adjal, M. Moussaoui, and A. Zine. Existence and regularity of solutions for stokes systems with non-smooth boundary data in a polyhedron. Electronic Journal of Differential Equations, 2017(147):1-10, 2017.

[4] T. Apel, S. Nicaise, and J. Pfefferer. Discretization of the Poisson equation with non-smooth data and emphasis on non-convex domains. Numer. Methods Partial Differential Equations, 32(5):1433-1454, 2016.

[5] T. Apel, S. Nicaise, and J. Pfefferer. Adapted numerical methods for the numerical solution of the poisson equation with 12 boundary data in non-convex domains. SIAM J. Numer. Anal., 55:1937-1957, 2017.

[6] D. Boffi, F. Brezzi, and M. Fortin. Mixed finite element methods and applications, volume 44 of Springer Series in Computational Mathematics. Springer, Heidelberg, 2013.

[7] K. Bogdan and B. Dyda. The best constant in a fractional hardy inequality. Math. Nachr., 284(5,6):629-638, 2011. 
[8] S. C. Brenner and L. R. Scott. The mathematical theory of finite element methods, volume 15 of Texts in Applied Mathematics. Springer, New York, third edition, 2008.

[9] Z. Cai and Y. Wang. An error estimate for two-dimensional Stokes driven cavity flow. Math. Comp., 78(266):771-787, 2009.

[10] C. Carstensen. Quasi-interpolation and a posteriori error analysis in finite element methods. M2AN Math. Model. Numer. Anal., 33(6):1187-1202, 1999.

[11] A. Cotsiolis and N. K. Tavoularis. Best constants for Sobolev inequalities for higher order fractional derivatives. J. Math. Anal. Appl., 295(1):225-236, 2004.

[12] W. Dahmen, B. Faermann, I. G. Graham, W. Hackbusch, and S. A. Sauter. Inverse inequalities on non-quasi-uniform meshes and the application to the mortar element method. Mathematics of Computation, 73(247):1107-1138, 2003.

[13] M. Dauge. Stationary Stokes and Navier-Stokes systems on two- or three-dimensional domains with corners. I. Linearized equations. SIAM J. Math. Anal., 20(1):74-97, 1989.

[14] E. B. Fabes, C. E. Kenig, and G. C. Verchota. The Dirichlet problem for the Stokes system on Lipschitz domains. Duke Math. J., 57(3):769-793, 1988.

[15] M. Feischl. Optimal adaptivity for a standard finite element method for the Stokes problem. ArXiv e-prints, arXiv:1710.08289, 2017.

[16] M. Hamouda, R. Temam, and L. Zhang. Modeling the lid driven flow: theory and computation. Int. J. Numer. Anal. Model., 14(3):313-341, 2017.

[17] R. B. Kellogg and J. E. Osborn. A regularity result for the Stokes problem in a convex polygon. Journal of Functional Analysis, 21:397-431, 1976.

[18] J.-L. Lions and E. Magenes. Non-homogeneous boundary value problems and applications. Vol. I. Springer-Verlag, New York-Heidelberg, 1972. Translated from the French by P. Kenneth, Die Grundlehren der mathematischen Wissenschaften, Band 181.

[19] M. Moussaoui and A. Zine. Existence and regularity results for the stokes system with non smooth boundary data in a polygon. Math. Models and Methods in Applied Sciences, 8(8):1307-1315, 1998.

[20] K. Schumacher. Solutions to the equation $\operatorname{div} u=f$ in weighted Sobolev spaces. In Parabolic and Navier-Stokes equations. Part 2, volume 81 of Banach Center Publ., pages 433-440. Polish Acad. Sci. Inst. Math., Warsaw, 2008.

[21] R. Temam. Navier-Stokes equations, volume 2 of Studies in Mathematics and its Applications. North-Holland Publishing Co., Amsterdam-New York, revised edition, 1979. Theory and numerical analysis, With an appendix by F. Thomasset.

[22] G. Verchota. Layer potentials and regularity for the Dirichlet problem for Laplace's equation in Lipschitz domains. Journal of Functional Analysis, 59:572-611, 1984.

[23] R. Verfürth. A posteriori error estimators for the Stokes equations. Numer. Math., 55(3):309$325,1989$.

Departamento de Matemática, Facultad de Ciencias Exactas y Naturales, Universidad de Buenos Aires and ImAs, COniCet, 1428 Buenos Aires, Argentina

E-mail address: rduran@dm.uba.ar

URL: http://mate.dm.uba.ar/〜rduran/

DiCATAM, Università di Brescia, Italy

E-mail address: lucia.gastaldi@unibs.it

$U R L:$ http://lucia-gastaldi.unibs.it

Departamento de Matemática, Facultad de Ciencias Exactas, Ingeniería y Agrimensura, Universidad Nacional de Rosario, and COniCet, Av. Pellegrini 250, 2000 Rosario, Argentina

E-mail address: ariel@fceia.unr.edu.ar

URL: https://fceia.unr.edu.ar/〜ariel/ 